\title{
1 Activin Signaling Regulates Autophagy and Cardiac Aging through mTORC2
}

2 Kai Chang ${ }^{1 \S}$, Ping Kang ${ }^{1 \S}$, Ying Liu ${ }^{1}$, Kerui Huang ${ }^{1}$, Erika Taylor ${ }^{2}$, Antonia P. Sagona ${ }^{3}$, Ioannis

3 P. Nezis ${ }^{3}$, Rolf Bodmer ${ }^{2}$, Karen Ocorr ${ }^{2}, \mathrm{Hua} \mathrm{Bai}^{1 *}$

51 Department of Genetics, Development, and Cell Biology, Iowa State University, Ames, IA

6 50011, USA

72 Development, Aging, and Regeneration Program, Sanford-Burnham-Prebys Medical

8 Discovery Institute, La Jolla, California 92037, USA

93 School of Life Sciences, University of Warwick, Coventry CV4 7AL, UK

11 *Corresponding Author:

12 Hua Bai

13 Phone: 515-294-9395

14 Email: hbai@iastate.edu

$16 \S$ These authors contribute equally to this work.

\section{Running title:}

22 Activin promotes cardiac aging through mTORC2 


\section{Abstract}

33 Age-dependent loss of cardiac tissue homeostasis largely impacts heart performance and

34 contributes significantly to cardiovascular diseases later in life. Cellular quality control

35 machinery, such as autophagy/lysosome system, plays a crucial role in maintaining cardiac

36 health and preventing age-induced cardiomyopathy and heart failure. However, how aging alters

37 the autophagy/lysosome system to impact cardiac function remains largely unknown. Here using

38 Drosophila heart as a model system, we show that activin signaling, a member of TGF-beta

39 superfamily, negatively regulates cardiac autophagy and cardiac health during aging. We found

40 that cardiac-specific knockdown of Daw, an activin-like protein in Drosophila, increased cardiac

41 autophagy and prevented age-related cardiac dysfunction, including arrhythmia and bradycardia

42 (slow heart rate). Inhibition of autophagy blocked Daw knockdown-mediated cardioprotection.

43 Consistently, cardiac-specific expression of constitutively activated activin type I receptor Babo

44 disrupted cardiac function at young ages. Intriguingly, the key autophagy regulator, mechanistic

45 target of rapamycin complex 1 (mTORC1), was not involved in activin-mediated autophagy.

46 Instead, activin signaling genetically interacted with Rictor, the key subunit of mTORC2, to

47 regulate autophagy and cardiac aging. Knockdown of Daw increased the mRNA expression of

48 Rictor and the phosphorylation of AKT in fly hearts. Finally, cardiac-specific silencing of Daw

49 not only improved cardiac health, but also prolonged lifespan. Thus, our findings highlight an

50 emerging role of activin signaling and mTORC2 in the regulation of autophagy and cardiac

51 aging.

53 Key words: Activin ligand, Dawdle, Atg8a, TSC1, Rictor, mTOR complex 2, Autophagic flux,

54 Semi-automatic optical heartbeat analysis (SOHA) 


\section{Introduction}

Aging is associated with an exponential increase of the incidence of cardiovascular diseases (CVD) ${ }^{1,2}$. Age-related changes in cardiovascular structure and output have been linked to increased risk of coronary heart disease, sudden cardiac death and stroke in elderly population

3. During normal aging, the left ventricular wall of human hearts becomes thickened and the diastolic filling rate of left ventricle gradually decreases. On the other hand, the left ventricular systolic performance at rest remains no change with age ${ }^{3}$. Several mechanisms underlying these age-associated changes in cardiovascular structure and function have been proposed, for example changes in growth factor signaling, decreased cellular quality control, altered calcium handling, elevated extracellular matrix deposition or fibrosis, increased mitochondria damage, and the production of reactive oxygen species (ROS) ${ }^{2,4}$. Resolving the contributing mechanisms underlying age-dependent decline of cardiovascular function is critical for the development of therapeutic interventions for the treatment of cardiovascular diseases.

Cellular quality control systems, such as macroautophagy (hereinafter referred to as autophagy), are essential to the maintenance of tissue homeostasis during aging ${ }^{5,6}$. Autophagy is a highly conserved process that is responsible for the degradation and recycling of damaged organelles, protein aggregates and other cytoplasmic substances. The autophagic process initiated with the isolated membrane, or phagophore that elongates to form a double-membrane structure, the autophagosome. Then the autophagosome fuses with a lysosome to form an autolysosome to degrade the cargo within the autolysosome ${ }^{7}$. It is generally accepted that autophagy activity declines with age ${ }^{8-10}$. Disruption of autophagy pathways often leads to loss of tissue homeostasis and tissue dysfunction. For example, knocking out autophagy gene Atg5 in the mouse heart accelerates cardiac aging, including an increase in left ventricular hypertrophy and decrease in 86 factional shortening ${ }^{10}$. As a crucial autophagy regulator, mechanistic target of rapamycin (mTOR),

87 especially mTOR complex 1 (mTORC1), plays an important role in the regulation of cardiac tissue homeostasis ${ }^{2}$. Rapamycin-mediated suppression of mTORC1 induces autophagy and protects mouse cardiomyocytes from oxidative stress ${ }^{11}$. In Drosophila, mTORC1 is known to promote electrical pacing-induced heart failure ${ }^{12,13}$, and high-fat-diet-induced cardiomyopathy ${ }^{14}$. The

91 mTOR signaling consists of two complexes, mTORC1 and mTORC2. It is not known whether

92 mTORC2 also contributes to the regulation of autophagy ${ }^{15}$. Although inhibition of mTOR is one

93 of the robust longevity interventions 16,17 , it remains unclear whether mTOR pathway plays a 
94 major role in age-dependent decline of autophagy. Notably, a recent study showed that mTORC1

95 activity does not increase with age in most tissues of C57BL/6J.Nia mice ${ }^{18}$. Thus, the mechanistic

96 basis for age-dependent alterations in autophagy and the contribution of mTORC1 remains to be

97 determined.

98 Autophagy can be activated by various stresses, such as dietary restriction, hypoxia, 99 oxidative stress, and pathogen infection ${ }^{19}$. The activation of autophagy is tightly controlled by key 100 cellular signaling pathways, in particular the nutrient signaling pathways like mTORC1 and AMP101 activated protein kinase (AMPK) ${ }^{20}$. These pathways are often involved in control of longevity.

102 We and other groups recently found that activin signaling, a transforming growth factor beta (TGF-

103 beta) subfamily, regulates muscle proteostasis and longevity in Drosophila ${ }^{21,22}$. As in mammals,

104 TGF-beta family in Drosophila has two major branches, bone morphogenetic protein (BMP) and 105 activin signaling pathways ${ }^{23}$. In both pathways, signaling starts with ligand binding to a receptor 106 complex composed of type I and type II receptor kinases, followed by phosphorylation of receptor107 activated Smad (R-Smad). Drosophila activin signaling is known to regulate neuroblast 108 proliferation, axon guidance, and metabolic homeostasis during development (reviewed in ${ }^{24}$ ). The 109 regulation of adult physiology by Drosophila activin is not well understood. We previously 110 showed that knockdown of one of the three activin ligands, Daw, prolongs lifespan and increases 111 autophagosome formation in flies ${ }^{21}$. However, the role of TGF-beta/activin signaling in cardiac 112 aging remains largely unknown.

113 It has been shown that TGF-beta factors promote cardiac fibrosis during aging ${ }^{25}$. Mice 114 with heterozygous mutants for activin receptor-like kinases (ALK4) are protected from pressure 115 overload-induced fibrosis and dysfunction ${ }^{26,27}$. Inhibition of ALK5 also reduces myocardial 116 infarction-induced systolic dysfunction and left ventricular remodeling in rats ${ }^{28}$. However, 117 conflicting results are reported in other studies showing that activin A protects hearts from 118 hypoxia/reoxygenation- and ischemia/reperfusion-induced cell death ${ }^{29}$. In addition, it is unknown 119 whether TGF-beta family proteins, in particular activin, regulate cardiac homeostasis and function 120 through autophagy. Given that the cardiac development and aging are remarkably conserved 121 between Drosophila and mammals, we investigated the role of activin signaling in the regulation 122 of cardiac autophagy and cardiac aging using Drosophila heart model and cardiac movement 123 detection techniques ${ }^{30}$. Here we show that activin signaling acts on hearts to regulate autophagy 124 and age-related cardiomyopathy in Drosophila. RNAi-mediated knockdown of the activin-like 
125 protein Daw preserved cardiac function with age, while expression of activated activin type I

126 receptor Babo induced cardiomyopathy early in life. Inhibition of autophagy blocked the

127 cardioprotective effects by Daw knockdown. Intriguingly, activin signaling genetically interacted

128 with mTORC2 subunit Rictor (rapamycin-insensitive companion of mTOR), but not mTORC1 to

129 control autophagy and cardiac aging. Thus, our study uncovered a novel interaction between

130 activin and mTORC2 in the regulation of tissue homeostasis, autophagy, and age-related

131 cardiomyopathy.

133 Results

134 Heart-specific knockdown of activin-like ligand Daw slows cardiac aging

135 Our previous study demonstrated that the activin-like protein Daw regulated tissue

136 homeostasis (especially in flight muscle) and longevity in Drosophila ${ }^{21}$. Interestingly, we found

137 that activin signaling increased in aging fly hearts, as indicated by elevated phosphorylation of

138 Smad2 in heart nuclei (Fig. 1a). This finding suggest that activin signaling may play a role in

139 regulating cardiac aging. To investigate this possibility, we knocked down the expression of Daw

140 specifically in the heart with a binary GAL4/UAS system where we crossed UAS-Daw RNAi

141 lines into a cardiac-specific tissue driver (Hand-gal4). We analyzed cardiac performance in

142 young and old flies using a high-speed video imaging system (semi-automatic optical heartbeat

143 analysis, SOHA) ${ }^{31}$. M-Mode traces from the SOHA analysis indicated that knockdown of Daw

144 preserved cardiac contractility at advanced ages (Fig. 1b). In wild-type flies, normal aging is

145 associated with increased cardiac arrhythmia, diastolic intervals, heart period (bradycardia, slow

146 heart rate) (Figs. 1c, 1d, 1e), and decreased cardiac output (Fig. 1f). Systolic intervals normally

147 remain unchanged (Fig. 1g). Interestingly, cardiac-specific knockdown of Daw attenuated age-

148 dependent increase in arrhythmia, diastolic intervals, and heart period (Figs. 1c, 1d, 1e). Daw

149 knockdown also maintained relatively normal cardiac output at advanced ages (Fig. 1f). Similar

150 results were observed from three independent Daw RNAi lines (the knockdown efficiency of

151 Daw RNAi was verified by qRT-PCR in our previous studies ${ }^{21}$ ).

152 The Hand-gal4 line drives gene expression in both cardiomyocytes and pericardial cells

$153 \quad 32$. To test the tissue-specific role of Daw in cardiac aging, we first crossed Daw RNAi into a

154 cardiomyocyte-specific driver TinC-gal4 ${ }^{33}$. Similar to the results from Hand-gal4 (Fig. 1h),

155 cardiomyocyte-specific knockdown of Daw prevented age-related increase in cardiac arrhythmia 
156 (Fig. 1i). We also tested whether Daw expression in pericardial cells can regulate cardiac aging using a pericardial cell-specific driver Dot-gal4 ${ }^{34}$. Interestingly, knockdown of Daw in pericardial cells also attenuated age-induced arrhythmia (Fig. 1j), but not the increase in diastolic interval (Fig. 1k). Thus, Daw appears to promote cardiac aging, in particular age-induced arrhythmia, through both cardiomyocyte-specific regulation and non-autonomous signaling from pericardial cells.

\section{Cardiomyocyte-specific knockdown of activin receptor Babo delays cardiac aging}

In Drosophila, activin-like ligand Daw signals through the type I receptor Babo, the fly homolog of mammalian ALK4 (activin receptor type-1B) ${ }^{23}$. To investigate whether Daw acts through its receptor Babo to modulate cardiac aging, we first asked if Babo plays a similar role in preventing age-dependent increase in cardiomyopathy. Consistent with the results from Daw knockdown, we found that cardiomyocyte-specific knockdown of Babo blocked the age-related increase in cardiac arrhythmia, diastolic intervals, and heart period (Figs. 2a, 2b, 2c). Two independent Babo RNAi lines were used and both gave similar results. Systolic intervals remained unchanged in both controls and Babo RNAi flies (Fig. 2d). Knockdown of Babo did not affect age-dependent changes in cardiac output (Data not shown).

175 hearts. We found that cardiac expression of activated Babo significantly increased cardiac

176 arrhythmia, diastolic intervals, and heart period already at young ages (Figs. 2e, 2f, 2g). Taken

177 together, these results suggest that Babo plays an important role in age-related cardiomyopathy, 178 especially cardiac arrhythmia, diastolic function and heart rate.

\section{Daw negatively regulates autophagy in fly hearts}

Autophagy is one of the key mechanisms in maintaining tissue hemostasis, and its

182 activity normally declines with age ${ }^{6,9,10}$. Our previous studies showed that activin signaling

183 regulates autophagosome formation and Atg8a transcription ${ }^{21}$. It is likely that Daw regulates

184 cardiac aging by modulating autophagy. To first verify the role of Daw in autophagy-lysosome

185 activity, we used LysoTracker staining to monitor the lysosomal acidification in adult fat body, a 186 well-established autophagy marker in flies ${ }^{35}$. Consistent with our previous finding, heterozygous 
$187 D^{2} w^{[1]}$ loss-of-function mutants showed increased LysoTracker staining, suggesting an elevated 188 basal autophagy/lysosome activities (Fig. 3a). Using a lipidation assay, we furthermore showed 189 that mutation of Daw increased the levels of lipidated Atg8a, the fly homolog of mammalian 190 LC3/GABARAP family proteins (Fig. 3b). Finally, mosaic analysis revealed that somatic cell 191 clones in larval fat body expressing RNAi against either Daw or Babo (marked by GFP) showed 192 increased numbers of autophagosome, indicated by mCherry-Atg8a puncta (Fig. 3c). The data 193 suggest that activin signaling negatively regulates autophagy and lysosome activities. Next, we examined whether Daw plays a similar role in modulating autophagy in fly 195 hearts. Using a commercial antibody for Drosophila Atg8a protein (Fig. S1 shows the antibody 196 validation), we found that the number of autophagosome in the heart was higher in Daw ${ }^{[11]}$ 197 mutants compared to wild-type (Fig. S2a). Because autophagy is a dynamic process, the 198 increased autophagosome number could result from increased autophagy activities, or blockage 199 of lysosomal degradation ${ }^{36}$. We next examined the role of Daw in the regulation of autophagic 200 flux (the turnover of autophagosome). We exposed semi-intact fly hearts to a V-ATPase inhibitor 201 bafilomycin A1 (Baf A1), which blocks both lysosomal acidification and autophagosome202 lysosome fusion ${ }^{37}$. We found that Baf A1 treatment resulted in a higher increase in the number 203 of Atg8a-positive autophagosome in $\mathrm{Daw}^{[11]}$ hearts compared to wild-type hearts (Figs. S2a204 S2c). Thus, the results suggest that Daw negatively regulate autophagic flux in fly hearts. Aging tissues show reduced autophagy and accumulated cellular damages. We next 206 examined whether inhibition of activin/Daw signaling in fly hearts can rescue age-dependent 207 decline of autophagy. Using a mCherry-Atg8a reporter line, we observed a dramatic 208 accumulation of Atg8a-positive autophagosome in the heart (Fig. 3d, 3e, 3h). The 209 cardiomyocyte-specific driver TinC-gal4 was used. The accumulation of autophagosome is 210 likely due to the blockage of autophagosome turnover, because increases numbers of Atg8a211 positive puncta were only seen in Baf A1-treated young hearts, but not the old hearts (Figs. 3e, $2123 \mathrm{~h}, 3 \mathrm{j}$ ). Consistently, the adaptor protein p62/Ref(2)P, a marker for defective aggrephagy ${ }^{38}$, was 213 accumulated in aging hearts and Atg8a mutants (Fig. S3). Intriguingly, flies with heart-specific

214 Daw knockdown maintained high levels of cardiac autophagosome turnover (or active 215 autophagic flux) at both young and old ages, as indicated by the induction of autophagosome 216 number upon Baf A1 treatment (Figs. 3f, 3h, 3j). Notably, constitutively activated Babo 217 repressed the autophagic flux at both young and old ages (Figs. 3g, 3h). Activation of Babo also 
blocked the accumulation of autophagosome in old fly hearts, which suggests that besides the regulation of autophagosome turnover, activin signaling also play an important role in autophagosome initiation and formation (Figs. 3g, 3h). We were able to verify these phenotypes using another heart-specific driver Hand-Gal4 (Fig. 3i). Taken together, our findings suggest that inhibition of activin/Daw signaling promotes autophagosome turnover and maintains healthy autophagic activity in old hearts.

\section{Inhibition of autophagy, but not activation of mTORC1, blocks Daw knockdown-mediated} cardioprotection

Since we observed an induction of autophagy in Daw knockdown flies, we next asked whether autophagy activity is required for Daw-regulated cardiac aging. First, we fed control and Daw knockdown flies with lysosomal inhibitor chloroquine (CQ). As expected, CQ treatment increased cardiac arrhythmia in Daw knockdown flies at old age (Fig. 4a). To directly test whether autophagy plays any role in Daw-regulated cardiac aging, we generated double knockdown flies by combining UAS-Daw RNAi and UAS-Atg1 RNAi fly lines. Atg1 is a serine/threonine-protein kinase that is essential for autophagy initiation. Cardiac-specific knockdown of Atgl increased both cardiac arrhythmia and diastolic intervals at young ages (Fig. 4b), similar to the fly heart with activated Babo (Figs. 2e, 2f). Consistent with CQ treatment, cardiac-specific knockdown of Atgl attenuated cardioprotective effects of Daw RNAi, since simultaneously knockdown of Atgl and Daw in the heart led to age-dependent increase in arrhythmia similar to control flies (Fig. 4c). Thus, these results suggest that autophagy and Atg1 are required for Daw-regulated cardiac aging.

mTORC1 is a key negative regulator of autophagy ${ }^{35}$. We then tested whether Daw could negatively regulate autophagy and cardiac aging through activation of mTORC1 signaling. To test this possibility, we first generated double knockdown flies by combining UAS-Daw RNAi and UAS-Tsc1 RNAi. Tsc1 (Tuberous sclerosis protein 1) is a negative regulator of mTORC1 (Fig. S4a). Surprisingly, activation of mTORC1 via Tsc1 knockdown had no effects on cardiac arrhythmia (Fig. 4d), but reduced diastolic intervals at young age (Fig. 4e), which is very different from the cardiomyopathy resulted from cardiac-specific expression of activated activin receptor Babo (Figs. 2e, 2f). Furthermore, knockdown of Tscl did not rescue the cardioprotective effects of Daw knockdown during aging (Fig. 4f). Simultaneously knockdown 
of Tsc1 and Daw did not show age-dependent increase in arrhythmia. Similarly, loss-of-function mutant $T s c 1^{[12]}$ did not rescue the elevated LysoTracker staining in $D a w^{[11]}$ (Fig. 4g). These results suggest that activation of $\mathrm{mTORC1}$ is not required for Daw-regulated autophagy and cardiac aging.

It is known that inhibition of mTORC1 prolongs lifespan ${ }^{16,39}$ and attenuates stressinduced cardiomyopathies ${ }^{12,13,40,41}$. However, the effects of mTORC1 induction on cardiac aging are not well understood. Here, we observed unique changes of cardiac function in response to activation of mTORC1 (via Tscl knockdown), including decreased heart period (Tachycardia, fast heart rate), and reduced diastolic intervals (Figs. 4d, 4e) These cardiac functional changes are not the same as age-induced cardiomyopathies, rather they resemble high-fat-diet-induced cardiac complications where mTORC1 is known to play an important role ${ }^{14}$. Intriguingly, flies with cardiac-specific knockdown of Tscl showed no age-dependent increases in heart period, 261 diastolic intervals, and arrhythmia (Figs. S4b, S4c, S4d). These unique alterations in cardiac function were also observed in other mTORC1 activation mutants, such as 1) Knockdown of Reptor (Repressed by TOR), a recently identified bZIP transcription factor that is negatively regulated by mTORC1 ${ }^{42}$ (Figs. S4e, S4f, S4g), and 2) Over-expression of Rheb (Ras Homolog Enriched in Brain), a positive regulator of mTORC1 (Figs. S4h, S4i, S4j).

\section{Daw genetically interacts with mTORC2/Rictor to control autophagy and cardiac aging}

Although Daw did not interact mTORC1 to regulate autophagy and cardiac aging, cardiac-specific knockdown of Daw induced the phosphorylation of AKT, the upstream regulator of mTORC1 (Figs. 5a, 5b). On the other hand, activated receptor Babo reduced cardiac AKT

272 kinases at distinct positions, PDK1 (phosphoinositide-dependent kinase 1) at Thr308 and Rictor 273 (major component of mTORC2) at Ser473 (Ser505 in flies) ${ }^{43}$. Cardiac-specific over-expression

274 Rictor can also induce AKT phosphorylation (Figs. 5e, 5f). Interestingly, knockdown of Daw up275 regulated the mRNA expression of Rictor in fly hearts (Figs. 5g, 5h). These results suggest that Daw is a negative regulator of $\mathrm{mTORC} 2 /$ Rictor.

Unlike mTORC1, the role of mTORC2 in the regulation of autophagy is unknown.

278 Intriguingly, we found that loss-of-function mutation in Rictor repressed the high lysosomal 279 activities in $\mathrm{Daw}^{[11]}$ mutants (Figs. 5i, 5j, 5k, 51). Similarly, mutations in Sin1, another core 
component of mTORC2, blocked the elevated lysosomal activity in Daw mutants. Furthermore, mosaic analysis revealed that over-expression of Rictor induced lysosome activity (Fig. 5m). Interestingly, unlike $T s c 1^{[12]}$ mutants, Rictor $^{[42]}$ mutants did not repress starvation-induced

283 lysosome activity (Fig. S5c). Similarly, activated Babo also did not block starvation-induced

284 lysosome activity (Fig. S5d). These data suggest that activin signaling and mTORC2/Rictor regulate autophagy differently from $\mathrm{mTORC} 1$, and Rictor acts downstream of activin signaling in the regulation of autophagy.

Given that Rictor positively regulates autophagy, we predict that Rictor is a cardiac protective factor. Indeed, cardiac-specific over-expression of Rictor blocked age-related increases in diastolic intervals and arrhythmia (Figs. 6a, 6b), while knockdown of Rictor induced arrhythmia, especially at old ages (Fig. 6c). Interestingly, over-expression of Rictor attenuated

291 the elevated diastolic intervals and arrhythmia in fly hearts with constitutively activated Babo

292 (Figs. 6d, 6e), suggesting that fly activin signaling regulates cardiac aging through

293 mTORC2/Rictor (Fig. 6f).

\section{Cardiac-specific reduction of activin-like protein Daw prolongs lifespan}

Tissue-specific hormonal signaling in systemic aging control have been previously reported $^{8,44,45}$. However, whether longevity can be achieved by maintaining healthy hearts remains unclear. Here we tested the effects of cardiac-specific knockdown of activin signaling in longevity control. Knockdown of Daw in the heart using two cardiac tissue drivers (Hand-gal4 and TinC-gal4) significantly prolongs lifespan (range from 14\% to $32 \%$ extension of median lifespan) (Figs. 7a-7e). Compared to Hand-gal4, cardiomyocyte-specific driver TinC-gal4 produced stronger lifespan extension. Two independent lifespan analyses were performed and both showed similar lifespan phenotypes (Fig. 7e). In the first lifespan trial, multiple control lines (dashed lines in Fig. 7a) and two Daw RNAi lines (solid lines in Fig. 7a) were included. Because Daw is a hormonal factor, it is unclear whether Daw regulates lifespan through cellautonomous or non-autonomous mechanisms. However, our previous study showed that Daw regulates longevity in a tissue-specific manner ${ }^{21}$. Flight muscle-specific knockdown of Daw prolongs lifespan, while fat body-specific knockdown of Daw shortens lifespan. Therefore, it is possible that cardiac-specific Daw signaling regulates longevity through maintenance of cardiac health and production of unknown systemic factors from the fly heart. 


\section{Discussion}

313 TGF-beta signaling plays vital roles in a wide range of human diseases, including cancer

314 and cardiovascular diseases ${ }^{46}$. Originally identified as a reproductive hormone, activin, a TGF-

315 beta subfamily member, has become an emerging target for the treatment of many human

316 diseases ${ }^{47}$. In the present study, we investigated the role of activin in cardiac aging using a

317 Drosophila heart model. Similar to our previous study in Drosophila flight muscle ${ }^{21}$, reduction

318 of activin signaling prevented age-related decline of cardiac function. We think that this is partly

319 due to the activation of autophagy. Daw mutant flies exhibited high autophagic flux and elevated

320 lysosome activities. Genetic analysis further showed that autophagy gene Atg1 is required for

321 Daw-regulated cardiac aging. Importantly, we found that activation of mTORC1 did not rescue

322 the autophagy and cardiac aging phenotypes in Daw knockdown flies. Instead, activin/Daw

323 signaling interacts with mTORC2 subunit Rictor to regulate autophagy and cardiac function.

324 Thus, our studies uncover an emerging role of activin signaling and mTORC2 in the regulation

325 of autophagy and cardiac tissue homeostasis.

326 Although previous studies reveal an interesting link between TGF-beta family proteins

327 and animal aging ${ }^{21,48-50}$, the role of activin in cardiac aging is not well understood. It's known

328 that activin A serum levels significantly increase with age ${ }^{51}$. The mRNA expression of activin A,

329 as well as serum activin A levels are positively correlated with heart failure ${ }^{52,53}$, and

330 hypertension in the elderly ${ }^{54}$. These findings suggest that activation of activin signaling at old

331 ages might be detrimental to the heart. Studies showed that activin A promotes cardiac fibrosis

332 and myocardial damage after ischemia reperfusion ${ }^{55}$. Consistently, mice with heterozygous

333 mutations of ALK4 are protected from pressure overload-induced fibrosis and dysfunction ${ }^{26,27 .}$

334 Inhibition of ALK5 also reduced myocardial infarction-induced systolic dysfunction and left

335 ventricular remodeling in rats ${ }^{28}$. However, other studies suggest that activin A protects hearts

336 from hypoxia/reoxygenation- and ischemia/reperfusion-induced cell death ${ }^{29}$, even though it can

337 promote cardiac apoptosis at higher concentrations ${ }^{56}$. The findings from the present study

338 support the negative regulatory role of activin signaling in cardiac function, in particular age-

339 related cardiac dysfunction (e.g., arrhythmia). This is consistent with the pro-aging role of TGF-

340 beta and activin ${ }^{21,50}$. 
Activin signaling has been implicated in the regulation of a wide range of cellular

342 processes, including cell death and proliferation, inflammation, fibrosis, and metabolic

343 homeostasis ${ }^{47,57-59}$. The role of activin signaling in the control of autophagy, a key tissue

344 maintenance process, was only identified recently ${ }^{21,60-63}$. In C. elegans, reduction of activin-like

345 protein Daf-7 suppressed beta-amyloid peptide-induced autophagosome accumulation ${ }^{62,63}$.

346 Another recent study found that activin A blocked oxygen-glucose deprivation-induced

347 autophagy through the inhibition of JNK and p38 MAPK pathways in neuronal PC12 cells ${ }^{60}$.

348 Our previous study showed that knockdown of Drosophila activin-like protein Daw in flight

349 muscle induced autophagosome formation and autophagy gene expression ${ }^{21}$. However, how

350 activin signaling regulates autophagy and how this regulation contributes to tissue homeostasis

351 during aging remain largely unknown. Our findings in the present study suggest that the fly

352 activin-like protein Daw promotes cardiac aging through inhibition of autophagy. It seems that

353 activin signaling controls both autophagosome formation and turnover. Importantly, fly hearts

354 with reduced Daw expression maintain high levels of autophagosome turnover (autophagic flux)

355 throughout the life, whereas the autophagosome turnover is largely blocked in aged hearts in

356 wild-type flies. The active autophagosome turnover (and degradation capacity) at old ages is

357 likely responsible for the healthy hearts seen in Daw knockdown flies.

358 mTORC1 is a well-known autophagy regulator and it inhibits autophagosome formation

359 by increasing the phosphorylation of the serine/threonine protein kinase Atg1 (ULK1/ULK2 in

360 mammals), the initiator of the autophagy machinery ${ }^{7}$. Intriguingly, activation of mTORC1 did

361 not block the induction of autophagosome formation in Daw knockdown flies, suggesting that

362 there might be unknown mTORC1-independent mechanisms involved in Daw-regulated

363 autophagy and cardiac aging. mTORC1-independent autophagy regulation has been observed in

364 several recent studies ${ }^{64-66}$. A genome-wide screen study identified an important role of type III

365 PI3 kinases in autophagy control under normal nutritional conditions ${ }^{64}$. The screen also revealed

366 that growth factor signaling pathways, such as MAPK-ERK and AKT/FOXO3, negatively

367 regulate autophagy by inhibiting the type III PI3 kinase cascade. Interestingly, we noticed that

368 knockdown of Daw led to elevated AKT phosphorylation and the expression of mTORC2

369 subunit Rictor, suggesting mTORC2/AKT signaling may be involved in Daw-regulated

370 autophagy and cardiac aging. Indeed, we found that mutation of Rictor blocked the activation of

371 autophagy in Daw knockdown flies. mTORC2 is one of the two mTOR complexes that is 
372 insensitive to short-term rapamycin treatment, and is involved in glucose homeostasis and actin 373 cytoskeleton reorganization ${ }^{67,68}$. The direct regulatory role of mTORC2/Rictor in cellular

374 autophagy has not been previously established, although a few studies suggest that Rictor may be 375 involved in autophagy activation under unique circumstances. For example, Rictor is required for 376 resveratrol-induced autophagy in rat myocardium and $\mathrm{H} 9 \mathrm{c} 2$ cardiac myoblast cells ${ }^{69}$. In rat 377 kidney NRK-52E cells, silencing Rictor blocked cisplatin induced autophagy ${ }^{70}$. Our findings 378 align with these studies suggesting that Rictor is a positive regulator of autophagy. However, 379 conflicting findings are reported in other studies showing Rictor negatively regulates 380 autophagosome formation in mouse skeletal muscle ${ }^{71}$, and LC3-II levels in human senescent 381 endothelial cells ${ }^{72}$. Thus, more studies are needed to better understand the role of 382 mTORC2/Rictor in the regulation of autophagy and its contribution to tissue homeostasis during 383 aging.

384 The positive role of Rictor in autophagy control identified in the present study is consistent with the cardioprotection effects of Rictor. We found that cardiac-specific overexpression of Rictor slows cardiac aging, similar to Daw knockdown. Our findings are consistent

387 with two recent studies showing that disruption of mammalian Rictor induced cardiac dysfunction ${ }^{73,74}$. Cardiac-specific deletion of Rictor in mice induced dilation and decreased fractional shortening by activating MST1 kinase and hippo pathway ${ }^{74}$. Unlike mTORC1 mutants, Rictor null mutants (in both worms and mice) are short-lived ${ }^{75,76}$, suggesting the

392 longevity regulation is not yet known, although it has been shown that Drosophila mutants of

393 Rictor and Sin 1 decreased the tolerance to heat stress ${ }^{77}$ and over-expression of Rictor rescued

394 Pink1 knockdown-induced mitochondrial aggregation in flight muscle ${ }^{78}$. The Rictor-Pink1

395 interaction suggests that Rictor could be a potential positive regulator for mitophagy, a key

396 process for the mitochondria quality control during aging.

397 During normal aging, the heart undergoes complex phenotypic changes such as

398 progressive myocardial remodeling, reduced myocardial contractile capacity, increased left

399 ventricular wall thickness and chamber size, prolonged diastole as well as increased arrhythmia

$400{ }^{3}$. All of these biological changes can gradually alter cardiac functions and confer vulnerability of

401 the heart to various cardiovascular stresses, thus increasing the chance of developing

402 cardiovascular disease dramatically. The present study has uncovered an important role of TGF- 
403 beta/activin signaling pathway in the regulation of autophagy and cardiac aging in Drosophila.

404 We also found an intriguing interaction between activin and mTORC2 in the regulation of 405 cardiac autophagy, as well as a new role of Rictor in cardiac aging in Drosophila. It remains to 406 be determined how activin regulates Rictor in fly hearts. It is possible that activin signaling 407 regulates Rictor through both transcriptional activation (e.g., through insulin/FOXO signaling ${ }^{79}$ ) 408 and post-translational modification.

409 Additionally, cardiac-specific knockdown of activin-like protein Daw extended fly 410 lifespan, suggesting that longevity can be achieved by maintaining a healthy heart. Our findings 411 also suggest that Daw itself or other unidentified hormonal factors secreted from cardiomyocytes 412 play an important role in systemic aging control. Hence, it remains to be determined the 413 mechanistic basis for activin-mediated control of cardiac aging and longevity, which will 414 eventually help the development of therapeutic interventions targeting activin for the treatment 415 of age-related cardiovascular diseases.

Materials and methods

Flies were maintained at $25^{\circ} \mathrm{C}, 60 \%$ relative humidity and 12-hour light/dark $(40 \%$ relative humidity was used in the experiments from Figures $1 \& 2$, which were performed in 421 Bodmer laboratory). Adults were reared on agar-based diet with $0.8 \%$ cornmeal, $10 \%$ sugar, and $4222.5 \%$ yeast (unless otherwise noted). Fly stocks used in the present study are: UAS-Daw RNAi 423 (Bloomington Drosophila Stock Center (BDSC) \#34974, BDSC \#50911, and Vienna Drosophila 424 Resource Center (VDRC) \#105309), UAS-Babo RNAi (BDSC \#25933, BDSC \#40866), UAS425 Babo-Act (also known as Babo.Q302D) ${ }^{80}$, UAS-Tsc1 RNAi (BDSC \#52931, \#54034), UAS426 Reptor RNAi (BDSC \#25983), UAS-Rheb (BDSC \#9688), UAS-Atg1 RNAi (BDSC \#26731), 427 UAS-Rictor RNAi (BDSC \#36699), Daw ${ }^{[11] 81}$, Tscl $^{[12] 82}$, Rictor $^{[42] ~ 83}$, Sinl $^{[e 03756]}$ (BDSC $^{\text {B }}$ 428 \#18188), UAS-Rictor ${ }^{77}$, UAS-HA-Rictor ${ }^{77}$, Hand-gal4 (also known as Hand4.2-gal4) ${ }^{32}$, and 429 TinC-gal4 (also known as TinC 44 -gal4) ${ }^{33}$, Dot-gal4 ${ }^{34}$, Hand-GS-gal4 ${ }^{84}$, UAS-mCherry-Atg8a 430 (BDSC \#37750), Atg8a ${ }^{\Delta 4} 85$.

432 (BDSC \#31114) were backcrossed into $y w^{R}$ background for 5 7 generations, and $y w^{R}$ flies were 433 used as control or wild-type (WT) flies in most of the experiments. For other UAS-RNAi lines 
434 that were not backcrossed to $y w^{R}$, following genotypes were used as control: $y^{l} s c^{*} v^{l}$;

435 P[VALIUM20-mCherry]attP2 (BDSC \#35785), $y^{l} v^{l}$; P[CaryP]attP2 (BDSC \#36303), or $y^{l} v^{l}$;

436 P[CaryP]attP40 (BDSC \# 36304). Female flies were used in all experiments. RU486

437 (mifepristone, Sigma, St. Louis, MO, USA) were used to activate Hand-GS-Gal4 at a final

438 concentration of $200 \mu \mathrm{M}$ mixed in food.

Mosaic analysis

Two FLPout lines were used: 1). hs-flp; endogenous P-3x mCherry-Atg8a, UASGFP/Cyo; Act>CD2>Gal4,UAS-Dcr2/TM6) (kindly provided by Gábor Juhász). 2). yw, hs-flp, UAS-CD8::GFP; Act >y+>Gal4,UAS-GFP.nls;UAS-Dcr2/SM6::TM6B (kindly provided by Jun-

444 yuan Ji, originally generated by Bruce Edgar). To generate clones, freshly laid eggs (within 4-6 $445 \mathrm{hr}$ ) were heated in a $37^{\circ} \mathrm{C}$ water bath for $45 \mathrm{~min}$. Early L3 larvae (84 hours after egg laying) 446 were used in the experiments.

Fly heartbeat analysis

To measure cardiac function parameters, semi-intact Drosophila adult fly hearts were prepared according to previously described protocols ${ }^{31}$. High-speed 3000 frames movies were taken at a rate of 100 frames per second using a Hamamatsu ORCA-Flash4.0 digital CMOS camera (Hamamatsu Photonics, Hamamatsu, Japan) on an Olympus BX51WI microscope with a 10X water immersion lens (Olympus, Waltham, MA, USA) (Hamamatsu EM-CCD 9300 camera

454 was used in the experiments from Figures 1\&2, which were performed in Bodmer laboratory).

455 The live images that contain heart tub within abdominal A3 segment were processed using HCI

456 imaging software (Hamamatsu Photonics, Hamamatsu, Japan). M-modes and cardiac parameters 457 were generated using SOHA, a MATLAB-based image application as described previously ${ }^{31,86}$.

458 The M-mode provides a snapshot of movement of heart wall over time. Four cardiac parameters 459 were analyzed, including heart period, diastolic interval, systolic interval, and arrhythmia index. 460 Diastolic interval (DI) is the duration for each heart relaxation phase (diastole). Heart period 461 (HP) is the pause time between the two consecutive diastole (HP is the reciprocal of heart rate). 462 Systolic interval (SI) was calculated as the HP minus the DI. Arrhythmia index (AI) is the 463 standard deviation of all HP in each fly normalized to the median HP. Cardiac output was 
464 calculated using following equation: $\left(\pi \mathrm{r}(\mathrm{d})^{2}-\pi \mathrm{r}(\mathrm{s})^{2}\right) \times \mathrm{HR} \cdot \mathrm{r}(\mathrm{d})$ is radius of the heart tube at 465 diastolic phase, while $\mathrm{r}(\mathrm{d})$ is radius of the heart tube at systolic phase.

\section{Quantitative RT-PCR}

RNA extraction and cDNA synthesis were performed using Cells-to-CT kit (Thermo Fisher Scientific, Waltham, MA, USA) from 15 dissected adult hearts. QRT-PCR was performed with a Quantstudio 3 Real-Time PCR System (Thermo Fisher Scientific, Waltham, MA, USA). mRNA abundance of each gene was normalized to the expression of ribosomal protein L32 ( $R p L 32$ or $r p 49$ ) by the method of comparative $\mathrm{C}_{\mathrm{T}}$. Primer sequences are listed in

473 Supplementary Table S1.

\section{Antibodies and immunostaining}

Since commercial antibodies for Drosophila Atg8a show non-specific staining in immunostaining of fly tissues (data not shown), we used a GABARAP (E1J4E) antibody (1:300)

478 (Cell Signaling Technology (CST) \#13733, Danvers, MA, USA) to stain and detect endogenous sequences to Drosophila Atg8a protein (personal communication with Cell Signaling Technology, and see sequence alignment in Fig. S1a). This antibody has been previously used in several Drosophila autophagy studies ${ }^{87-89}$, and verified by Kim et al. ${ }^{90}$. We further verified the specificity of the GABARAP antibody using an Atg8a loss-of-function mutant $\operatorname{Atg}^{8} a^{\Delta 4}$ (Figs.

484 S1b-S1d). Other antibodies used for immunostaining are list below: $\operatorname{Ref}(2) \mathrm{P}(1: 1000)^{38}$,

485 Phospho-Smad2 antibody (1:500) (CST \#3108), Phospho-Akt (Ser473) (1:300) (CST \# 4060),

486 Alexa Fluor 488-conjugated Phalloidin (or Alexa Fluor 594) for F-actin staining (Thermo Fisher

487 Scientific, Waltham, MA, USA). All fluorescence-conjugated secondary antibodies were from

488 Jackson ImmunoResearch, West Grove, PA, USA).

489 For immunostaining, adult female flies were collected and dissected in PBS. Hearts were

490 fixed in $4 \%$ paraformaldehyde for 15 min at room temperature (RT). After washing in PBS with

$4910.1 \%$ Triton X-100 (PBST), the fixed hearted were blocked in 5\% normal goat serum (NGS)

492 diluted in PBST for 1 hour at RT. Hearts were then washed with PBST and incubated overnight 493 at $4^{\circ} \mathrm{C}$ with primary antibodies diluted in 5\% NGS. After washing with PBST, the samples were 494 incubated for 2 hours at RT with appropriate fluorescence-conjugated secondary antibodies. 
495

Hearts were mounted in ProLong Gold anti-fade reagent (Thermo Fisher Scientific, Waltham, MA, USA) before imaged using an epifluorescence-equipped BX51WI microscope (Olympus, Waltham, MA, USA).

\section{Imaging analysis}

For imaging analysis, fluorescence images were first processed using the deconvolution module in Olympus cellSens Demensions software, and then the number of puncta in a selected perinuclear region $\left(\sim 707 \mu \mathrm{m}^{2}\right)$ was measured with the "Measure and Count" module in cellSens software (Olympus, Waltham, MA, USA).

\section{LysoTracker and Magic Red staining}

Acidic organelles (including autolysosome) were monitored by staining tissue with 100 nM LysoTracker Red DND-99 (Invitrogen, Grand Island, NY, USA) for 5 minutes at room temperature. Lysosomal cathepsin B activities were monitored using Magic Red Cathepsin-B Assay kit (\#938, ImmunoChemistry Technologies, Bloomington, MN, USA) following manufacturer's manual. Nuclei were stained with either DAPI or Hoechst $33342(1 \mu \mathrm{g} / \mathrm{ml})$ (ImmunoChemistry Technologies).

\section{Western blotting for Atg8a lipidation}

Antibodies for western blot included: $\beta$-actin antibody (1:2000) (CST \#4967), GABARAP (E1J4E) antibody (1:2000) (CST \#13733), and HRP-conjugated secondary antibodies (Jackson ImmunoResearch, West Grove, PA, USA). Flies were first transferred to centrifuge tube containing $1 x$ laemmli buffer ( $10 \mu \mathrm{l}$ buffer for each milligram of fly), and heated at $100^{\circ} \mathrm{C}$ for $3 \mathrm{~min}$. The samples were then homogenized and heated again at $100^{\circ} \mathrm{C}$ for $3 \mathrm{~min}$. After the centrifuge at 13000x rpm for 5 min, supernatants were collected and loaded onto MiniPROTEAN precast gels (Bio-Rad, Hercules, CA, USA). Following incubation with primary and secondary antibodies, the blots were visualized with Pierce ECL Western Blotting Substrate (Thermo Fisher Scientific, Waltham, MA, USA).

\section{Bafilomycin A1 and Chloroquine Treatment}


For Bafilomycin A1 treatment, semi-intact hearts were incubated with $100 \mathrm{nM}$ of bafilomycin A1 (Alfa Aesar, Haverhill, MA, USA) in artificial hemolymph (buffer receipt in ${ }^{31}$ ) for two hours at room temperature prior to the appropriate immunostaining. DMSO was used as a control.

For chloroquine treatments, $100 \mu \mathrm{l}$ of $20 \mathrm{mM}$ chloroquine diphosphate salt (SigmaAldrich, St Louis, MO, USA) was added onto the fly food. Flies were fed with chloroquine for at least 24 hours prior to the cardiac analysis or the western blots.

\section{Demography and survival analysis}

Newly enclosed female flies were allowed to mate for two days, then separated from males and assigned to replicate one-liter demography cages at a density of 100-125 flies per cage. Three independent cages were set-up per genotype. Food was changed every two days, at which time dead flies were removed from the cage and counted. Survival analysis was conducted with JMP statistical software (SAS Institute, Cary, NC, USA), and data from replicate cages were combined. Mortality distributions were compared by Log-rank test.

\section{Statistical analysis}

GraphPad Prism (GraphPad Software, La Jolla, CA) was used for statistical analysis. To compare the mean value of treatment groups versus that of control, either student t-test or oneway ANOVA was performed using Dunnett's test for multiple comparison. The effects of mutants during aging was analyzed by two-way ANOVA, including Tukey multiple comparisons test. In SOHA analysis, the outliers were identified using Robust regression and Outlier removal (ROUT) method $(\mathrm{Q}=1 \%)$ prior to the data analysis. 


\section{Acknowledgements}

557 We thank Bloomington Drosophila Stock Center, Harvard Medical School, and Drosophila

558 Genomics Resource Center for fly stocks and cDNA clones. We thank Michael O'Connor for

559 activin reagents and fly stocks, Duojia Pan, Stocker Hugo, Jongkyeong Chung, Gábor Juhász,

560 Jun-yuan Ji, Bruce Edgar for fly stocks. Marc Tatar for help with Demography and survival

561 analysis. This work was supported by NIH/NIA R00 AG048016 and AFAR Research Grants to

562 HB.

Author Contributions Statement

Conceived and designed the experiments: KC RB KO HB. Performed the experiments: KC PK

YL KH APS IPN HB. Analyzed the data: KC PK YL KH ET APS IPN HB. Wrote the paper: KC

RB KO HB. All authors reviewed and approved the manuscript.

\section{Competing Interests}

570 The authors declare that no competing interest exists.

\section{Figure Legends}

\section{Figure 1. Heart-specific knockdown of Daw slows cardiac aging.}

577 a. Immunostaining of phospho-Smad2 in fly hearts at young (2 weeks) and old ages (6 weeks).

578 Arrows indicate cardiomyocyte nuclei and positive pSmad2 staining. Scale bar: $20 \mu \mathrm{m}$.

579 b. Representative M-mode traces ( 8 seconds) showing age-dependent movement of heart wall in 580 control and heart-specific Daw knockdown flies (Hand-gal4>UAS-Daw ${ }^{R N A i}$ ).

581 c-g. Age-related changes in arrhythmia index, diastolic intervals, heart period, cardiac output, 582 and systolic intervals in control $(\mathrm{Ctrl})$ and cardiac-specific Daw knockdown flies ( $\left.\operatorname{Daw}^{R N A i}\right)$.

583 Flies were cultured at $40 \%$ relative humidity. Hand-gal4 driver was used to knock down gene 584 expression specifically in cardiac tissues (cardiomyocytes and pericardial cells). Results from 585 three independent $U A S-D a w^{R N A i}$ lines are shown (RNAi \#1: BDSC \#34974, RNAi \#2: VDRC 586 \#105309, RNAi \#3: BDSC \#50911). Statistical significance is assessed by two-way ANOVA 
followed by Tukey multiple comparisons test $(* \mathrm{p}<0.05$, ** $\mathrm{p}<0.01, * * * \mathrm{p}<0.001, \mathrm{~ns}=$ not significant). $\mathrm{N}=7 \sim 20$. The interaction between genotype and age is statistically significant for heart period $(\mathrm{P}=0.0041)$ and diastolic interval $(\mathrm{P}=0.0243)$.

h-j. Age-dependent changes in cardiac arrhythmia between control and Daw knockdown using various tissue drivers, Hand-gal4 (cardiomyocytes and pericardial cells), TinC-gal4 (cardiomyocytes), and Dot-gal4 (pericardial cells). Student t-test $(* \mathrm{p}<0.05$, ** $\mathrm{p}<0.01$, *** $\mathrm{p}<0.001) . \mathrm{N}=25 \sim 31$.

k. Age-dependent changes in diastolic intervals between control and Daw knockdown in pericardial cells (Dot-gal4). Student t-test $(* \mathrm{p}<0.05$, ** $\mathrm{p}<0.01, * * * \mathrm{p}<0.001) . \mathrm{N}=13 \sim 26$.

Figure 2. Cardiomyocyte-specific knockdown of activin receptor Babo delays cardiac aging.

a-d. Age-dependent changes in cardiac arrhythmia, diastolic intervals, heart period, and systolic intervals in control $(\mathrm{Ctrl})$ and cardiomyocyte-specific Babo knockdown flies $\left(B a b o^{R N A i}\right)$. Flies were cultured at $40 \%$ relative humidity. TinC-gal4 driver was used. Results from two independent $U A S-B a b o^{R N A i}$ lines are shown (RNAi \#1: BDSC \#25933, RNAi \#2: BDSC \#40866). $\mathrm{N}=15 \sim 30$.

603 e-g. Changes in cardiac arrhythmia, diastolic intervals and heart period in fly hearts expressing 604 constitutively activated Babo $\left(B a b o^{A c t}\right)$. GeneSwitch heart driver Hand-GS-gal4 was used to 605 induce adult-onset Babo activation. RU: RU486 (Mifepristone). Flies were cultured at 40\% 606 relative humidity. $\mathrm{N}=6 \sim 9$. One-way ANOVA $(* * * \mathrm{p}<0.001, * * \mathrm{p}<0.01, * \mathrm{p}<0.05$, ns $=$ not 607 significant).

\section{Figure 3. Daw negatively regulates autophagy in fly hearts.}

610 a. Representative images of LysoTracker staining of young adult fat body between wild-type 611 (WT) and heterozygous Daw ${ }^{[11]}$ mutants. Scale bar: $20 \mu \mathrm{m}$.

612 b. Western blot analysis on Atg8a lipidation between $W T$ and $D a w^{[11]}$ mutants. GABARAP 613 antibodies were used to detect Atg8a. The lower band represents lipidated Atg8a. Flies were fed 614 with chloroquine (CQ, $20 \mathrm{mM})$ for at least 24 hours prior to western blots.

615 c. Representative images of mosaic analysis on autophagosome staining in larval fat body. Fat 616 body clones were generated by crossing $D a w^{R N A i}$ and $B a b o^{R N A i}$ lines into FLPout line carrying 617 mCherry-Atg8a reporter (hs-flp; endogenous P-3x mCherry-Atg8a, UAS-GFP/Cyo; 
$619 \mu \mathrm{m}$.

620 d. Representative images of fly hearts expressing autophagosome reporter mCherry-Atg8a at

621 both young and old ages. Arrows indicate cardiomyocyte nuclei and surrounding

622 autophagosomes. Abdominal segments A2-A3 are shown. Heart tube is located between two

623 yellow dashed lines. PC: Pericardial cell.

624 e-g. Representative images of mCherry-Atg8a reporter surrounding cardiomyocyte nuclei in

625 control, $D a w^{R N A i}$, and $B a b o^{A c t}$ flies. Semi-intact hearts were incubated with $100 \mathrm{nM}$ of

626 bafilomycin A1 (Baf A1) for two hours prior to immunostaining. Scale bar: $10 \mu \mathrm{m}$.

627 h. Quantification of age-dependent changes in mCherry-Atg8a-positive puncta in perinuclear

628 region in control, Daw $w^{R N A i}$, and $B a b o^{A c t}$ flies. TinC-gal4 was used to drive the expression of

629 Atg8a reporter and gene knockdown. One-way ANOVA $(* * * \mathrm{p}<0.001, * * \mathrm{p}<0.01, * \mathrm{p}<0.05$, ns

$630=$ not significant). $\mathrm{N}=5 \sim 7$.

631 i. Quantification of age-dependent changes in mCherry-Atg8a-positive puncta in perinuclear

632 region in control and $D a w^{R N A i}$. Hand -gal4 was used to drive the expression of Atg8a reporter

633 and Daw knockdown. One-way ANOVA $(* * * \mathrm{p}<0.001, * * \mathrm{p}<0.01, * \mathrm{p}<0.05$, ns $=$ not

634 significant). $\mathrm{N}=5 \sim 7$.

635 j. Quantification of Baf A1-induced mCherry-Atg8a-positive puncta between control and

$636 D a w^{R N A i}$.

Figure 4. Inhibition of autophagy, but not activation of mTORC1, blocks Daw knockdownmediated cardioprotection.

641 knockdown flies (Hand-gal4 used). One-way ANOVA $(* * * \mathrm{p}<0.001, * * \mathrm{p}<0.01, * \mathrm{p}<0.05$, ns $=$ 642 not significant). $\mathrm{N}=14 \sim 38$.

643 b. Cardiac arrhythmia and diastolic intervals in heart-specific knockdown of Atg 1 at young ages

644 (2-week-old) (Hand-gal4 used). One-way ANOVA $(* * * \mathrm{p}<0.001, * * \mathrm{p}<0.01, * \mathrm{p}<0.05$, ns $=$ not

645 significant). $\mathrm{N}=16 \sim 19$.

646 c. Age-dependent changes in cardiac arrhythmia in control, Daw ${ }^{R N A i}$, and $\operatorname{Daw}^{R N A i} ; \operatorname{Atg}^{R N A i}$ flies

647 (TinC-gal4 used). One-way ANOVA (*** $\mathrm{p}<0.001, * * \mathrm{p}<0.01, * \mathrm{p}<0.05$, ns $=$ not significant).

$648 \quad \mathrm{~N}=16 \sim 35$. 
d-e. Cardiac arrhythmia and diastolic intervals of Tscl knockdown flies (TinC-gal4 used). Two independent Tsc1 RNAi lines were used (RNAi-1: BDSC \#52931, RNAi-2: BDSC \#54034). One-way ANOVA $(* * * \mathrm{p}<0.001, * * \mathrm{p}<0.01, * \mathrm{p}<0.05, \mathrm{~ns}=$ not significant $) . \mathrm{N}=16 \sim 18$.

f. Age-dependent changes in cardiac arrhythmia in control, $D a w^{R N A i}$, and $D a w^{R N A i} ; T s c 1^{R N A i}$ flies

653 (TinC-gal4 used). One-way ANOVA (*** $\mathrm{p}<0.001, * * \mathrm{p}<0.01, * \mathrm{p}<0.05$, ns = not significant).

$654 \mathrm{~N}=7 \sim 30$.

655 g. Representative images of LysoTracker staining in adult fat body of WT, Daw ${ }^{[11]}, T_{s c 1^{[12]}}$ and 656 double mutant Daw ${ }^{[11]} ;$ Tscl $^{[12]}$. Scale bar: $10 \mu \mathrm{m}$.

a. Representative images of phospho-AKT staining in cardiomyocytes of control and Daw

660 knockdown flies (Hand-gal4). Scale bar: $10 \mu \mathrm{m}$.

b. Image quantification of Figure 5a. Student t-test $(* \mathrm{p}<0.05$, ** $\mathrm{p}<0.01$, *** $\mathrm{p}<0.001)$. N=5.

c. Representative images of phospho-AKT staining in cardiomyocytes of control and Daw

663 knockdown flies (Hand-gal4). Scale bar: $10 \mu \mathrm{m}$.

664 d. Image quantification of Figure 5c. Student t-test $(* \mathrm{p}<0.05, * * \mathrm{p}<0.01, * * * \mathrm{p}<0.001)$. N=5.

665 e. Representative images of phospho-AKT staining in cardiomyocytes of control and Rictor 666 over-expression (Hand-gal4). Two independent Rictor over-expression used (\#1: UAS-Rictor, $667 \quad \# 2:$ UAS-HA-Rictor $\left.{ }^{77}\right)$. Scale bar: $10 \mu \mathrm{m}$.

668 f. Image quantification of Figure 5e. One-way ANOVA $(* * * \mathrm{p}<0.001, * * \mathrm{p}<0.01, * \mathrm{p}<0.05$, ns $=$ 669 not significant). $\mathrm{N}=5$.

670 g. QRT-PCR analysis of Rictor expression in Daw knockdown flies (Hand-gal4). Student t-test $671 \quad(* \mathrm{p}<0.05, * * \mathrm{p}<0.01, * * * \mathrm{p}<0.001) . \mathrm{N}=3$.

672 h. QRT-PCR analysis of Daw expression in Daw knockdown flies (Hand-gal4). Student t-test (* $673 \mathrm{p}<0.05, * * \mathrm{p}<0.01, * * * \mathrm{p}<0.001) . \mathrm{N}=3$.

674 i. Representative images of LysoTracker staining in adult fat body of WT, Daw ${ }^{[11]}$, Rictor $^{[42]}$ and 675 double mutant Rictor ${ }^{[42]} ;$ Daw $^{[11]}$. Scale bar: $20 \mu \mathrm{m}$.

676 j. Image quantification of Figure 5i. One-way ANOVA $(* * * \mathrm{p}<0.001, * * \mathrm{p}<0.01, * \mathrm{p}<0.05$, ns $=$ 677 not significant). $\mathrm{N}=5$.

678 k. Representative images of Magic Red staining in adult fat body of WT, Daw ${ }^{[11]}$, Rictor $^{[42]}$ and 679 double mutant Rictor $^{[42]} ;$ Daw $^{[11]}$. Scale bar: $20 \mu \mathrm{m}$. 
1. Image quantification of Figure 5k. One-way ANOVA $\left(* * * \mathrm{p}<0.001, * * \mathrm{p}<0.01,{ }^{*} \mathrm{p}<0.05\right.$, ns $=$ not significant). $\mathrm{N}=5$.

m. Representative images of mosaic analysis of LysoTracker staining in larval fat body. Fat body clones were generated by crossing Rictor overexpression lines into a FLPout line ( $y w$, $h s-f l p$, UAS-CD8::GFP;Act>y+>Gal4,UAS-GFP.nls;UAS-Dcr2). Clones with Rictor overexpression are GFP-positive cells (dashed lines). Scale bar: $20 \mu \mathrm{m}$.

Figure 6. Heart-specific overexpression of Rictor preserved cardiac function during aging.

a-b. Diastolic intervals and arrhythmia in flies with heart-specific overexpression of Rictor $\mathrm{N}=19 \sim 26$.

c. Cardiac arrhythmia in flies with heart-specific knockdown of Rictor. One-way ANOVA (*** $\mathrm{p}<0.001, * * \mathrm{p}<0.01, * \mathrm{p}<0.05, \mathrm{~ns}=$ not significant). $\mathrm{N}=24$.

d-e. Diastolic intervals and arrhythmia in flies with heart-specific expression of Babo ${ }^{A c t}$, or both $B a b o^{A c t}$ and Rictor (Hand-gal4). One-way ANOVA (*** $\mathrm{p}<0.001, * * \mathrm{p}<0.01, * \mathrm{p}<0.05$, ns $=$ not

694 significant). $\mathrm{N}=11 \sim 27$.

695 f. Proposed model for activin-mTORC2 interaction in the regulation of autophagy and cardiac 696 aging.

Figure 7. Cardiac-specific knockdown of Daw prolongs lifespan.

a-b. Survival analysis of cardiomyocyte-specific (TinC-gal4) knockdown of Daw. Two independent trials and multiple controls ( $y w x y w$, yw $x$ Daw RNAi, TinC x mCherry RNAi) were performed. Log-Rank test, $\mathrm{p}<0.001$. c-d. Survival analysis of heart-specific (Hand-gal4) knockdown of Daw. Two independent trials were performed. Log-Rank test, $\mathrm{p}<0.001$. 


\section{Supplemental file}

\section{Table S1. Primer list}

\section{Figure S1. Validation of GABARAP antibody.}

715 a. Multiple sequence alignment between Drosophila Atg8a and human GABARAP using

716 ClustalW (https://www.genome.jp/tools-bin/clustalw). The red box indicates the predicted

717 epitope sequences used in producing GABARAP antibody.

718 b. Representative images of GABARAP immunostaining in fly hearts of WT and $\operatorname{Atg} 8 a^{\Delta 4}$

719 mutants. Heart tube is located between two yellow dashed lines. The panels on the right are the

720 zoomed-in images. Scale bar: $20 \mu \mathrm{m}$.

721 c. Representative images of GABARAP immunostaining in adult fat body of WT and $\operatorname{Atg} 8 a^{\Delta 4}$

722 mutants. The panels on the right are the zoomed-in images. Scale bar: $20 \mu \mathrm{m}$.

723 d. Western blots testing GABARAP antibody using $A \operatorname{tg} 8 a^{\Delta 4}$ mutants.

Figure S2. Daw inhibits autophagy flux in the heart.

726 a. Representative images of GABARAP immunostaining in fly hearts of WT and $D a w^{[11]}$ mutants

727 treated with or without Baf A1. Heart tube is located between two yellow dashed lines. The

728 panels on the right are the zoomed-in images. PC: Pericardial cell. Scale bar: $20 \mu \mathrm{m}$.

729 b. Quantification of Figure S2. One-way ANOVA $(* * * \mathrm{p}<0.001, * * \mathrm{p}<0.01, * \mathrm{p}<0.05$, ns $=$ not

730 significant). $\mathrm{N}=5$.

731 c. Quantification of Baf A1-induced puncta between $W T$ and Daw ${ }^{[11]}$ mutants.

733 Figure S3. Aging increases the accumulation of $\operatorname{Ref}(2) P$ in the heart.

734 a-b. Representative images of $\operatorname{Ref}(2) \mathrm{P}$ in young and old hearts. Scale bar: $10 \mu \mathrm{m}$.

735 c. Representative images of $\operatorname{Ref}(2) \mathrm{P}$ in the heart of $A \operatorname{tg} 8 a^{\Delta 4}$ mutants. Scale bar: $10 \mu \mathrm{m}$.

Figure S4. Activation of mTORC1 alters heart period, but not arrhythmia.

738 a. Schematic diagram for PI3K/AKT/mTOR signaling pathway.

739 b-d. Heart period, diastolic intervals, and arrhythmia in control ( $C t r l)$ and cardiomyocyte-

740 specific Tscl knockdown flies (TinC-gal4). One-way ANOVA (*** p<0.001, ** p<0.01, *

$741 \mathrm{p}<0.05, \mathrm{~ns}=$ not significant). $\mathrm{N}=14 \sim 18$. 
742 e-g. Heart period, diastolic intervals, and arrhythmia in control ( $C t r l)$ and cardiomyocyte-specific

743 Reptor knockdown flies (TinC-gal4). One-way ANOVA $(* * * \mathrm{p}<0.001, * * \mathrm{p}<0.01, * \mathrm{p}<0.05$, ns

$744=$ not significant). $\mathrm{N}=13 \sim 28$.

745 h-j. Heart period, diastolic intervals, and arrhythmia in control (Ctrl) and flies overexpressing

746 Rheb (TinC-gal4). One-way ANOVA $(* * * \mathrm{p}<0.001, * * \mathrm{p}<0.01, * \mathrm{p}<0.05$, ns $=$ not significant).

$747 \quad \mathrm{~N}=21 \sim 31$.

748

749 Figure S5. Autophagy regulation by mTORC2.

750 a. Representative images of LysoTracker staining in adult fat body of WT, Daw ${ }^{[11]}$, $\operatorname{Sin} 1^{[e 03756]}$

751 and double mutant $\operatorname{Sin} 1^{[e 03756]} ;$ Daw $^{[11]}$. Scale bar: $20 \mu \mathrm{m}$.

752 b. Image quantification of Figure S5a. One-way ANOVA $(* * * \mathrm{p}<0.001, * * \mathrm{p}<0.01, * \mathrm{p}<0.05, \mathrm{~ns}$ $753=$ not significant). $\mathrm{N}=5$.

754 c. Representative images of LysoTracker staining in fed and starved WT, Tsc1 mutants, and

755 Rictor mutants. Scale bar: $20 \mu \mathrm{m}$.

756 d. Representative images of mosaic analysis on LysoTracker staining in larval fat body of

$757 B a b o^{A c t}$ flies upon starvation. Larval fat body clones were generated using a FLPout line $(y w, h s$ -

758 flp, UAS-CD8::GFP;Act>y+>Gal4,UAS-GFP.nls;UAS-Dcr2). Clones with Babo ${ }^{A c}$ expression

759 are GFP-positive cells (dashed lines). Scale bar: $20 \mu \mathrm{m}$. 


\section{References}

1. Dai, D.F., Chen, T., Johnson, S.C., Szeto, H. \& Rabinovitch, P.S. Cardiac aging: from molecular mechanisms to significance in human health and disease. Antioxidants \& redox signaling 16, 1492-1526 (2012).

779

2. North, B.J. \& Sinclair, D.A. The intersection between aging and cardiovascular disease.

780 Circulation research 110, 1097-1108 (2012).

781

782

783

784

785

786

3. Lakatta, E.G. \& Levy, D. Arterial and cardiac aging: major shareholders in cardiovascular disease enterprises: Part II: the aging heart in health: links to heart disease. Circulation 107, 346-354 (2003).

4. Dai, D.F. et al. Overexpression of catalase targeted to mitochondria attenuates murine cardiac aging. Circulation 119, 2789-2797 (2009).

5. Quarles, E.K. et al. Quality control systems in cardiac aging. Ageing research reviews 23, 101-115 (2015).

6. Rubinsztein, D.C., Marino, G. \& Kroemer, G. Autophagy and aging. Cell 146, 682-695 (2011).

7. Shibutani, S.T. \& Yoshimori, T. A current perspective of autophagosome biogenesis.

Cell research 24, 58-68 (2014).

8. Demontis, F. \& Perrimon, N. FOXO/4E-BP signaling in Drosophila muscles regulates organism-wide proteostasis during aging. Cell 143, 813-825 (2010).

9. Chang, J.T., Kumsta, C., Hellman, A.B., Adams, L.M. \& Hansen, M. Spatiotemporal regulation of autophagy during Caenorhabditis elegans aging. eLife 6 (2017).

10. Taneike, M. et al. Inhibition of autophagy in the heart induces age-related cardiomyopathy. Autophagy 6, 600-606 (2010).

11. Dutta, D., Xu, J., Kim, J.S., Dunn, W.A., Jr. \& Leeuwenburgh, C. Upregulated autophagy protects cardiomyocytes from oxidative stress-induced toxicity. Autophagy 9, 328-344 (2013).

12. Wessells, R. et al. d4eBP acts downstream of both dTOR and dFoxo to modulate cardiac functional aging in Drosophila. Aging cell 8, 542-552 (2009).

13. Luong, N. et al. Activated FOXO-mediated insulin resistance is blocked by reduction of TOR activity. Cell metabolism 4, 133-142 (2006).

14. Birse, R.T. et al. High-fat-diet-induced obesity and heart dysfunction are regulated by the TOR pathway in Drosophila. Cell metabolism 12, 533-544 (2010).

15. Saxton, R.A. \& Sabatini, D.M. mTOR Signaling in Growth, Metabolism, and Disease. Cell 168, 960-976 (2017).

16. Kaeberlein, M. et al. Regulation of yeast replicative life span by TOR and Sch9 in response to nutrients. Science 310, 1193-1196 (2005).

17. Lee, J.H. et al. Sestrin as a feedback inhibitor of TOR that prevents age-related pathologies. Science 327, 1223-1228 (2010).

18. Baar, E.L., Carbajal, K.A., Ong, I.M. \& Lamming, D.W. Sex- and tissue-specific changes in mTOR signaling with age in C57BL/6J mice. Aging cell 15, 155-166 (2016).

19. Kroemer, G., Marino, G. \& Levine, B. Autophagy and the integrated stress response. Molecular cell 40, 280-293 (2010).

20. He, C. \& Klionsky, D.J. Regulation mechanisms and signaling pathways of autophagy. Annual review of genetics 43, 67-93 (2009). 
21. Bai, H., Kang, P., Hernandez, A.M. \& Tatar, M. Activin signaling targeted by insulin/dFOXO regulates aging and muscle proteostasis in Drosophila. PLoS genetics 9 , e1003941 (2013).

22. Demontis, F., Patel, V.K., Swindell, W.R. \& Perrimon, N. Intertissue control of the nucleolus via a myokine-dependent longevity pathway. Cell reports 7, 1481-1494 (2014).

23. Upadhyay, A., Moss-Taylor, L., Kim, M.J., Ghosh, A.C. \& O'Connor, M.B. TGF-beta Family Signaling in Drosophila. Cold Spring Harbor perspectives in biology (2017).

24. Upadhyay, A., Moss-Taylor, L., Kim, M.J., Ghosh, A.C. \& O'Connor, M.B. TGF-beta Family Signaling in Drosophila. Cold Spring Harbor perspectives in biology 9 (2017).

25. Biernacka, A. \& Frangogiannis, N.G. Aging and Cardiac Fibrosis. Aging and disease 2, 158-173 (2011).

26. Li, C.Y. et al. Partial inhibition of activin receptor-like kinase 4 attenuates pressure overload-induced cardiac fibrosis and improves cardiac function. Journal of hypertension 34, 1766-1777 (2016).

27. Chen, Y.H. et al. Haplodeficiency of activin receptor-like kinase 4 alleviates myocardial infarction-induced cardiac fibrosis and preserves cardiac function. Journal of molecular and cellular cardiology 105, 1-11 (2017).

28. Tan, S.M., Zhang, Y., Connelly, K.A., Gilbert, R.E. \& Kelly, D.J. Targeted inhibition of activin receptor-like kinase 5 signaling attenuates cardiac dysfunction following myocardial infarction. American journal of physiology. Heart and circulatory physiology 298, H1415-1425 (2010).

29. Oshima, Y. et al. Activin A and follistatin-like 3 determine the susceptibility of heart to ischemic injury. Circulation 120, 1606-1615 (2009).

30. Ocorr, K., Vogler, G. \& Bodmer, R. Methods to assess Drosophila heart development, function and aging. Methods 68, 265-272 (2014).

31. Ocorr, K., Fink, M., Cammarato, A., Bernstein, S. \& Bodmer, R. Semi-automated Optical Heartbeat Analysis of small hearts. Journal of visualized experiments : JoVE (2009).

32. Han, Z. \& Olson, E.N. Hand is a direct target of Tinman and GATA factors during Drosophila cardiogenesis and hematopoiesis. Development 132, 3525-3536 (2005).

33. Lo, P.C. \& Frasch, M. A role for the COUP-TF-related gene seven-up in the diversification of cardioblast identities in the dorsal vessel of Drosophila. Mechanisms of development 104, 49-60 (2001).

34. Zhang, F., Zhao, Y. \& Han, Z. An in vivo functional analysis system for renal gene discovery in Drosophila pericardial nephrocytes. Journal of the American Society of Nephrology : JASN 24, 191-197 (2013).

35. Scott, R.C., Schuldiner, O. \& Neufeld, T.P. Role and regulation of starvation-induced autophagy in the Drosophila fat body. Developmental cell 7, 167-178 (2004).

36. Klionsky, D.J. et al. Guidelines for the use and interpretation of assays for monitoring autophagy (3rd edition). Autophagy 12, 1-222 (2016).

37. Mauvezin, C., Nagy, P., Juhasz, G. \& Neufeld, T.P. Autophagosome-lysosome fusion is independent of V-ATPase-mediated acidification. Nature communications 6, 7007 (2015).

38. Nezis, I.P. et al. $\operatorname{Ref}(2) \mathrm{P}$, the Drosophila melanogaster homologue of mammalian p62, is required for the formation of protein aggregates in adult brain. The Journal of cell biology 180, 1065-1071 (2008). 
863

864

865

866

867

868

869

870

871

872

873

874

875

876

877

878

879

880

881

882

883

884

885

886

887

888

889

890

891

892

893

894

895

896

897

898

899

900

901

902

903

904

905

906

907

908

39. Johnson, S.C., Rabinovitch, P.S. \& Kaeberlein, M. mTOR is a key modulator of ageing and age-related disease. Nature 493, 338-345 (2013).

40. Shioi, T. et al. Rapamycin attenuates load-induced cardiac hypertrophy in mice. Circulation 107, 1664-1670 (2003).

41. Ding, Y. et al. Haploinsufficiency of target of rapamycin attenuates cardiomyopathies in adult zebrafish. Circulation research 109, 658-669 (2011).

42. Tiebe, M. et al. REPTOR and REPTOR-BP Regulate Organismal Metabolism and Transcription Downstream of TORC1. Developmental cell 33, 272-284 (2015).

43. Sarbassov, D.D., Guertin, D.A., Ali, S.M. \& Sabatini, D.M. Phosphorylation and regulation of Akt/PKB by the rictor-mTOR complex. Science 307, 1098-1101 (2005).

44. Hwangbo, D.S., Gershman, B., Tu, M.P., Palmer, M. \& Tatar, M. Drosophila dFOXO controls lifespan and regulates insulin signalling in brain and fat body. Nature 429, 562566 (2004).

45. Zhang, G. et al. Hypothalamic programming of systemic ageing involving IKK-beta, NFkappaB and GnRH. Nature 497, 211-216 (2013).

46. Akhurst, R.J. \& Hata, A. Targeting the TGFbeta signalling pathway in disease. Nature reviews. Drug discovery 11, 790-811 (2012).

47. Tsuchida, K. et al. Activin signaling as an emerging target for therapeutic interventions. Cell communication and signaling : CCS 7, 15 (2009).

48. Luo, S., Kleemann, G.A., Ashraf, J.M., Shaw, W.M. \& Murphy, C.T. TGF-beta and insulin signaling regulate reproductive aging via oocyte and germline quality maintenance. Cell 143, 299-312 (2010).

49. Narasimhan, S.D. et al. PDP-1 links the TGF-beta and IIS pathways to regulate longevity, development, and metabolism. PLoS genetics 7, e1001377 (2011).

50. Shaw, W.M., Luo, S., Landis, J., Ashraf, J. \& Murphy, C.T. The C. elegans TGF-beta Dauer pathway regulates longevity via insulin signaling. Current biology : $C B$ 17, 1635 1645 (2007).

51. Baccarelli, A. et al. Activin A serum levels and aging of the pituitary-gonadal axis: a cross-sectional study in middle-aged and elderly healthy subjects. Experimental gerontology 36, 1403-1412 (2001).

52. Yndestad, A. et al. Elevated levels of activin A in heart failure: potential role in myocardial remodeling. Circulation 109, 1379-1385 (2004).

53. Wei, Q. et al. The expression and role of activin A and follistatin in heart failure rats after myocardial infarction. International journal of cardiology 168, 2994-2997 (2013).

54. Tsai, Y.L. et al. The Association Between Serum Activin A Levels and Hypertension in the Elderly: A Cross-Sectional Analysis From I-Lan Longitudinal Aging Study. American journal of hypertension (2017).

55. Chen, Y. et al. Regulation and actions of activin A and follistatin in myocardial ischaemia-reperfusion injury. Cytokine 69, 255-262 (2014).

56. Liu, M., Mao, C., Li, J., Han, F. \& Yang, P. Effects of the Activin A-Follistatin System on Myocardial Cell Apoptosis through the Endoplasmic Reticulum Stress Pathway in Heart Failure. International journal of molecular sciences 18 (2017).

57. Ghosh, A.C. \& O'Connor, M.B. Systemic Activin signaling independently regulates sugar homeostasis, cellular metabolism, and $\mathrm{pH}$ balance in Drosophila melanogaster. Proceedings of the National Academy of Sciences of the United States of America 111, 5729-5734 (2014). 
909

910

911

912

913

914

915

916

917

918

919

920

921

922

923

924

925

926

927

928

929

930

931

932

933

934

935

936

937

938

939

940

941

942

943

944

945

946

947

948

949

950

951

952

953

954

58. Chng, W.B., Bou Sleiman, M.S., Schupfer, F. \& Lemaitre, B. Transforming growth factor beta/activin signaling functions as a sugar-sensing feedback loop to regulate digestive enzyme expression. Cell reports 9, 336-348 (2014).

59. Werner, S. \& Alzheimer, C. Roles of activin in tissue repair, fibrosis, and inflammatory disease. Cytokine \& growth factor reviews 17, 157-171 (2006).

60. Xue, L.X. et al. Activin A/Smads signaling pathway negatively regulates Oxygen Glucose Deprivation-induced autophagy via suppression of JNK and p38 MAPK pathways in neuronal PC12 cells. Biochemical and biophysical research communications 480, 355-361 (2016).

61. Guo, B. et al. Genome-wide screen identifies signaling pathways that regulate autophagy during Caenorhabditis elegans development. EMBO reports 15, 705-713 (2014).

62. Florez-McClure, M.L. \& Link, C.D. Erratum: "Florez-McClure ML, Hohsfield LA, Fonte G, Bealor MT, Link CD. Decreased insulin-receptor signaling promotes the autophagic degradation of beta-amyloid peptide in C. elegans". Autophagy 2007; 3:569-80. Autophagy 5, 132-133 (2009).

63. Florez-McClure, M.L., Hohsfield, L.A., Fonte, G., Bealor, M.T. \& Link, C.D. Decreased insulin-receptor signaling promotes the autophagic degradation of beta-amyloid peptide in C. elegans. Autophagy 3, 569-580 (2007).

64. Lipinski, M.M. et al. A genome-wide siRNA screen reveals multiple mTORC1 independent signaling pathways regulating autophagy under normal nutritional conditions. Developmental cell 18, 1041-1052 (2010).

65. Li, Y. et al. Protein kinase C controls lysosome biogenesis independently of mTORC1. Nature cell biology 18, 1065-1077 (2016).

66. Henson, S.M. et al. p38 signaling inhibits mTORC1-independent autophagy in senescent human CD8(+) T cells. The Journal of clinical investigation 124, 4004-4016 (2014).

67. Oh, W.J. \& Jacinto, E. mTOR complex 2 signaling and functions. Cell cycle 10, 23052316 (2011).

68. Gaubitz, C., Prouteau, M., Kusmider, B. \& Loewith, R. TORC2 Structure and Function. Trends in biochemical sciences 41, 532-545 (2016).

69. Gurusamy, N. et al. Cardioprotection by resveratrol: a novel mechanism via autophagy involving the mTORC2 pathway. Cardiovascular research 86, 103-112 (2010).

70. Li, J. et al. Rictor/mTORC2 protects against cisplatin-induced tubular cell death and acute kidney injury. Kidney international 86, 86-102 (2014).

71. Mammucari, C. et al. FoxO3 controls autophagy in skeletal muscle in vivo. Cell metabolism 6, 458-471 (2007).

72. Xiong, Y. et al. ARG2 impairs endothelial autophagy through regulation of MTOR and PRKAA/AMPK signaling in advanced atherosclerosis. Autophagy 10, 2223-2238 (2014).

73. Shende, P. et al. Cardiac mTOR complex 2 preserves ventricular function in pressureoverload hypertrophy. Cardiovascular research 109, 103-114 (2016).

74. Sciarretta, S. et al. mTORC2 regulates cardiac response to stress by inhibiting MST1. Cell reports 11, 125-136 (2015).

75. Soukas, A.A., Kane, E.A., Carr, C.E., Melo, J.A. \& Ruvkun, G. Rictor/TORC2 regulates fat metabolism, feeding, growth, and life span in Caenorhabditis elegans. Genes \& development 23, 496-511 (2009).

76. Lamming, D.W. et al. Depletion of Rictor, an essential protein component of mTORC2, decreases male lifespan. Aging cell 13, 911-917 (2014). 
955

956

957

958

959

960

961

962

963

964

965

966

967

968

969

970

971

972

973

974

975

976

977

978

979

980

981

982

983

984

985

986

987

988

989

77. Jevtov, I. et al. TORC2 mediates the heat stress response in Drosophila by promoting the formation of stress granules. Journal of cell science 128, 2497-2508 (2015).

78. $\mathrm{Wu}, \mathrm{Z}$. et al. Tricornered/NDR kinase signaling mediates PINK1-directed mitochondrial quality control and tissue maintenance. Genes \& development 27, 157-162 (2013).

79. Chen, C.C. et al. FoxOs inhibit mTORC1 and activate Akt by inducing the expression of Sestrin3 and Rictor. Developmental cell 18, 592-604 (2010).

80. Brummel, T. et al. The Drosophila activin receptor baboon signals through $\mathrm{dSmad} 2$ and controls cell proliferation but not patterning during larval development. Genes \& development 13, 98-111 (1999).

81. Serpe, M. \& O'Connor, M.B. The metalloprotease tolloid-related and its TGF-beta-like substrate Dawdle regulate Drosophila motoneuron axon guidance. Development 133, 4969-4979 (2006).

82. Gao, X. \& Pan, D. TSC1 and TSC2 tumor suppressors antagonize insulin signaling in cell growth. Genes \& development 15, 1383-1392 (2001).

83. Lee, G. \& Chung, J. Discrete functions of rictor and raptor in cell growth regulation in Drosophila. Biochemical and biophysical research communications 357, 1154-1159 (2007).

84. Monnier, V. et al. dJun and Vri/dNFIL3 are major regulators of cardiac aging in Drosophila. PLoS genetics 8, e1003081 (2012).

85. Pircs, K. et al. Advantages and limitations of different p62-based assays for estimating autophagic activity in Drosophila. PloS one 7, e44214 (2012).

86. Fink, M. et al. A new method for detection and quantification of heartbeat parameters in Drosophila, zebrafish, and embryonic mouse hearts. BioTechniques 46, 101-113 (2009).

87. Ratliff, E.P., Barekat, A., Lipinski, M.M. \& Finley, K.D. Brain trauma and autophagy: What flies and mice can teach us about conserved responses. Autophagy 12, 2256-2257 (2016).

88. Ratliff, E.P. et al. Assessing Basal and Acute Autophagic Responses in the Adult Drosophila Nervous System: The Impact of Gender, Genetics and Diet on Endogenous Pathway Profiles. PloS one 11, e0164239 (2016).

89. Barekat, A. et al. Using Drosophila as an integrated model to study mild repetitive traumatic brain injury. Scientific reports 6, 25252 (2016).

90. Kim, M. et al. Drosophila Gyf/GRB10 interacting GYF protein is an autophagy regulator that controls neuron and muscle homeostasis. Autophagy 11, 1358-1372 (2015). 
Figure 1
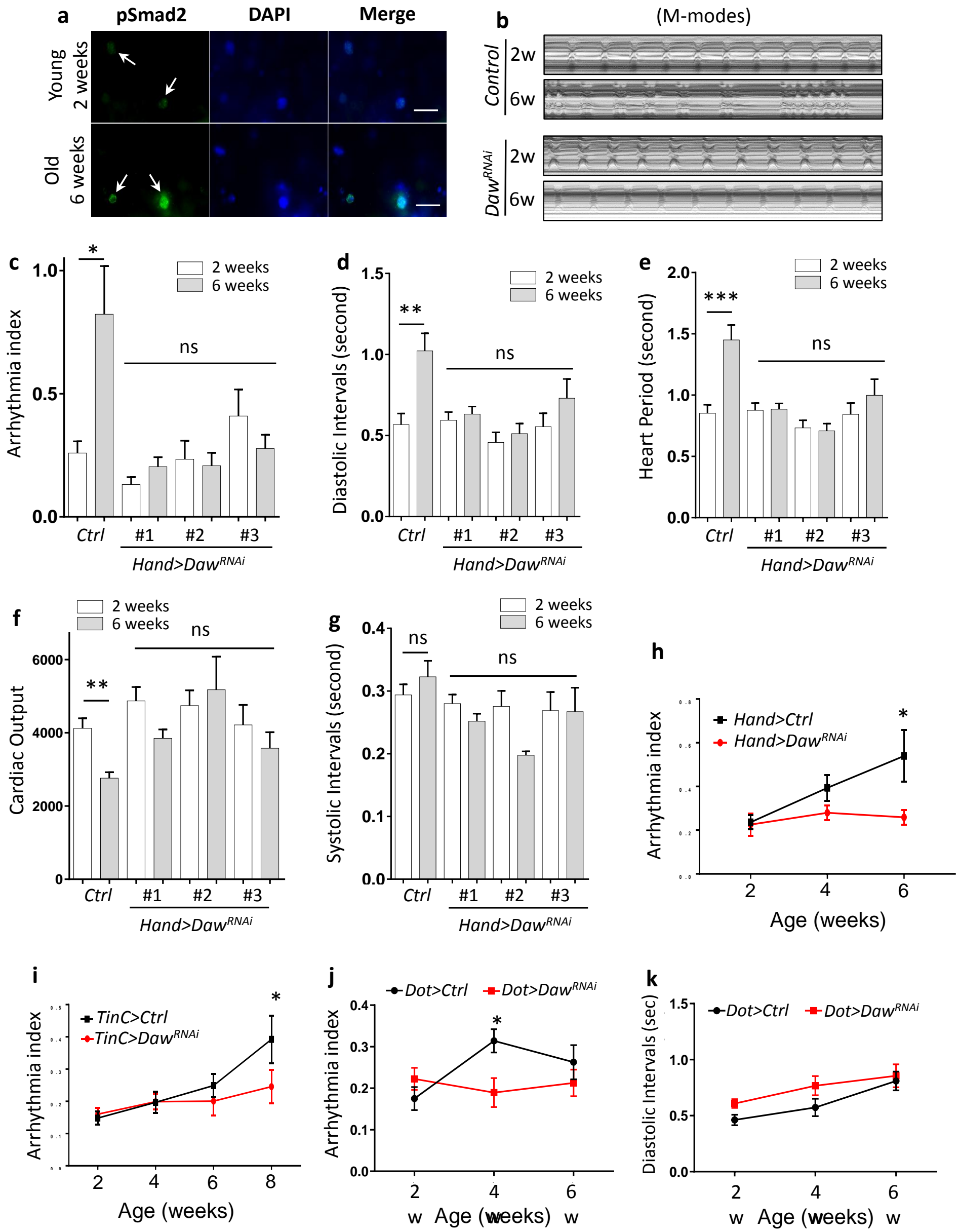
Figure 2
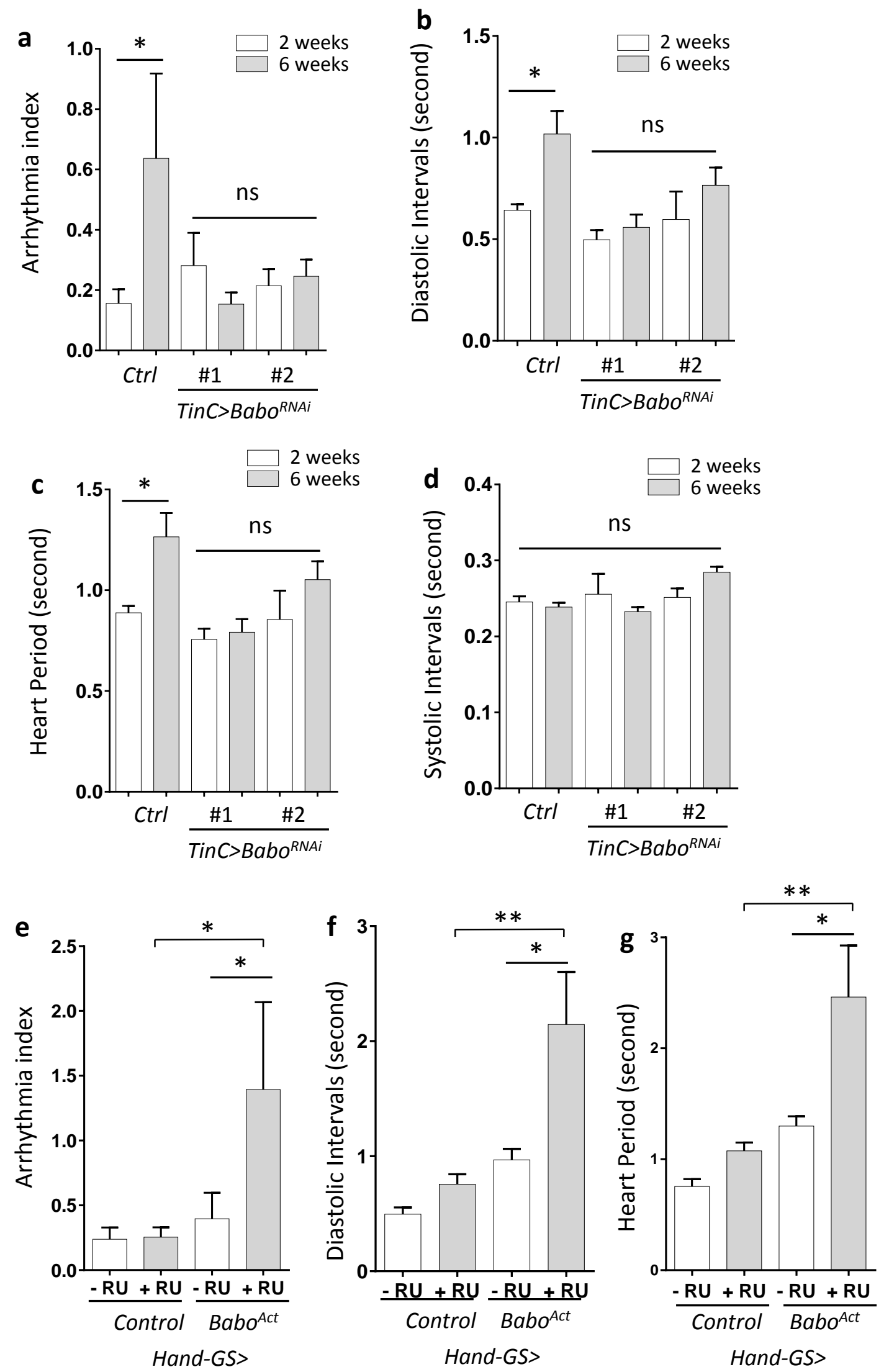
Figure 3

a

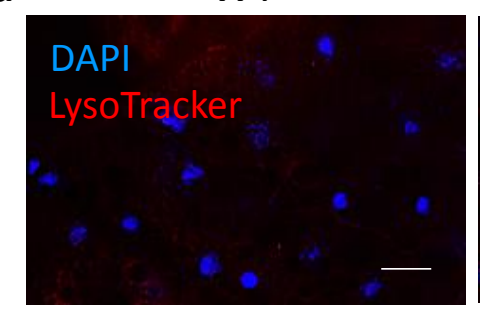

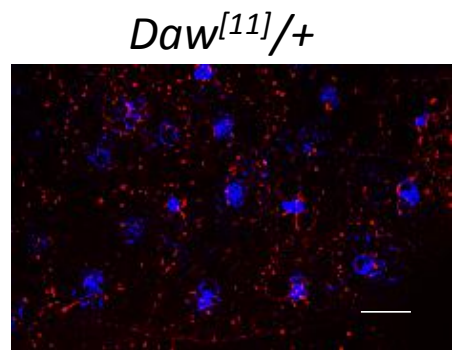

b $\mathrm{CQ}$
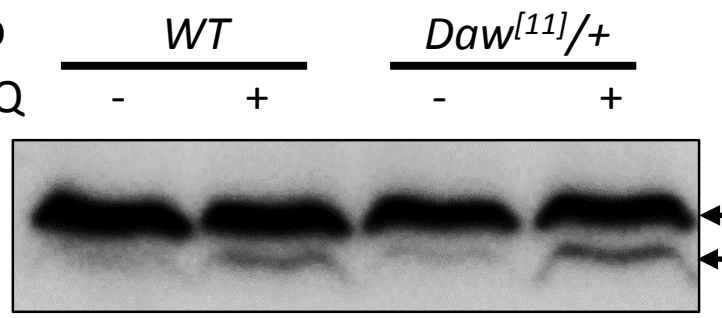

Merge

C
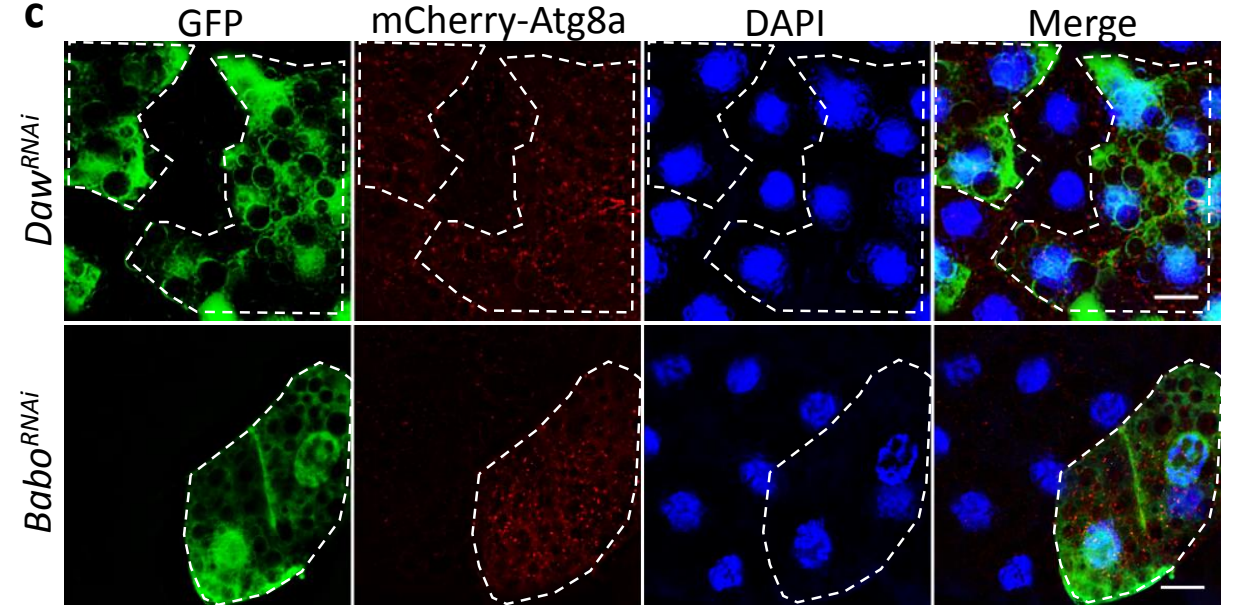

d
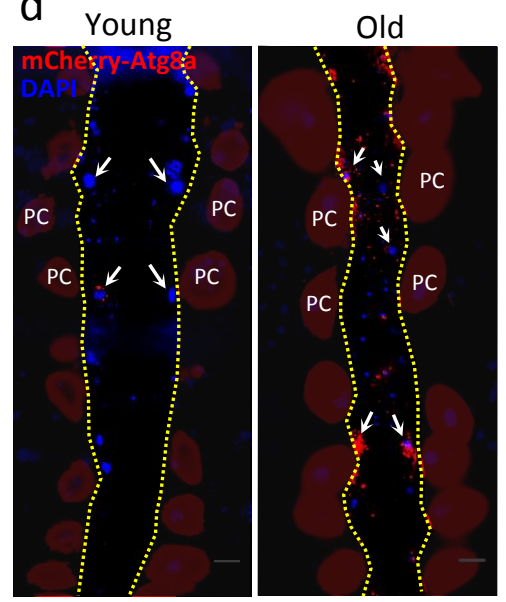

g

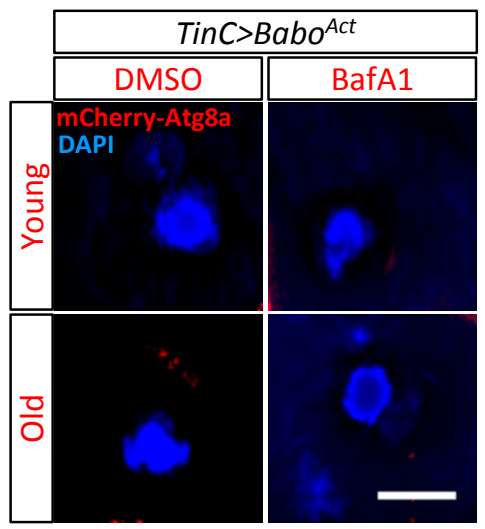

e

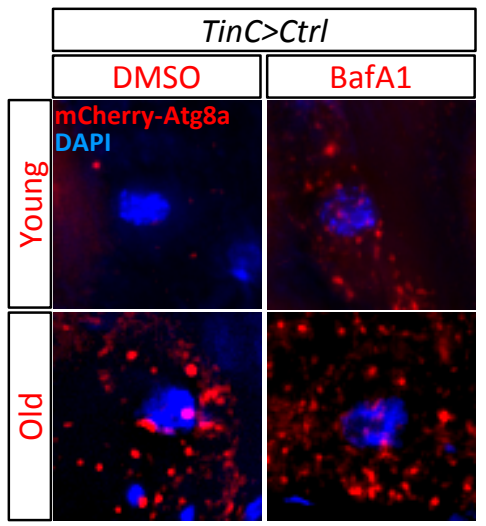

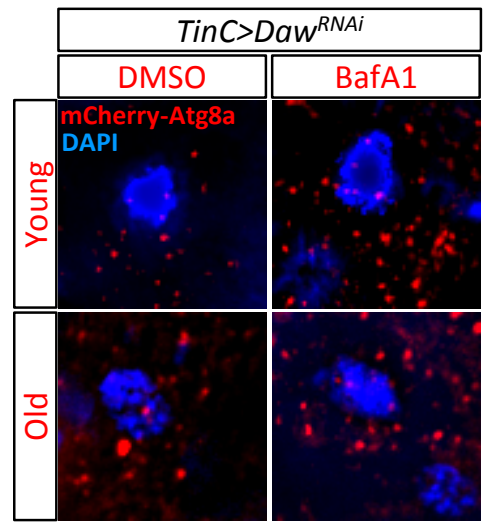

f
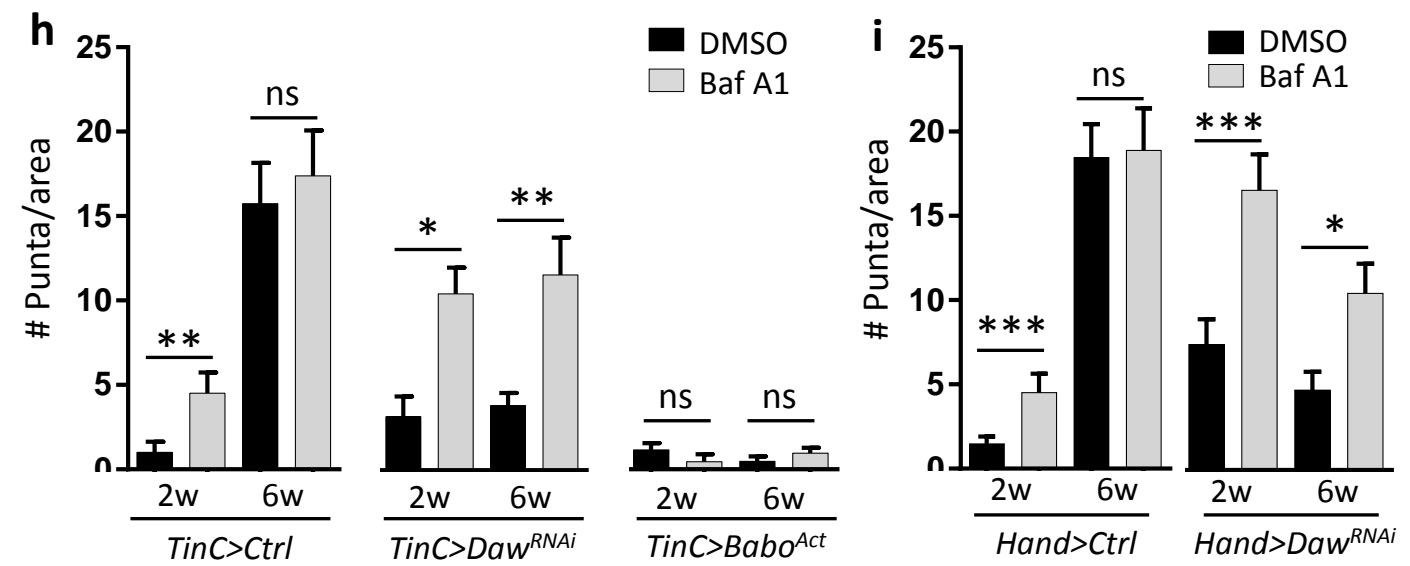

-Atg8a-I

-Atg8a-II j

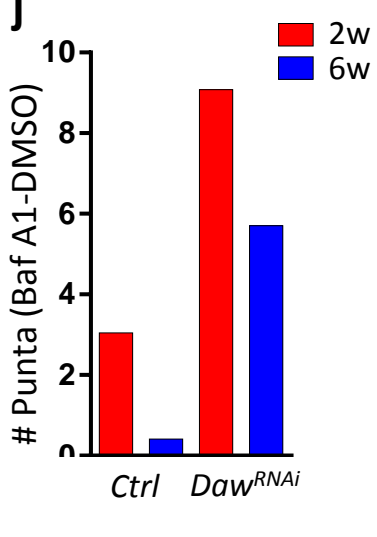


Figure 4
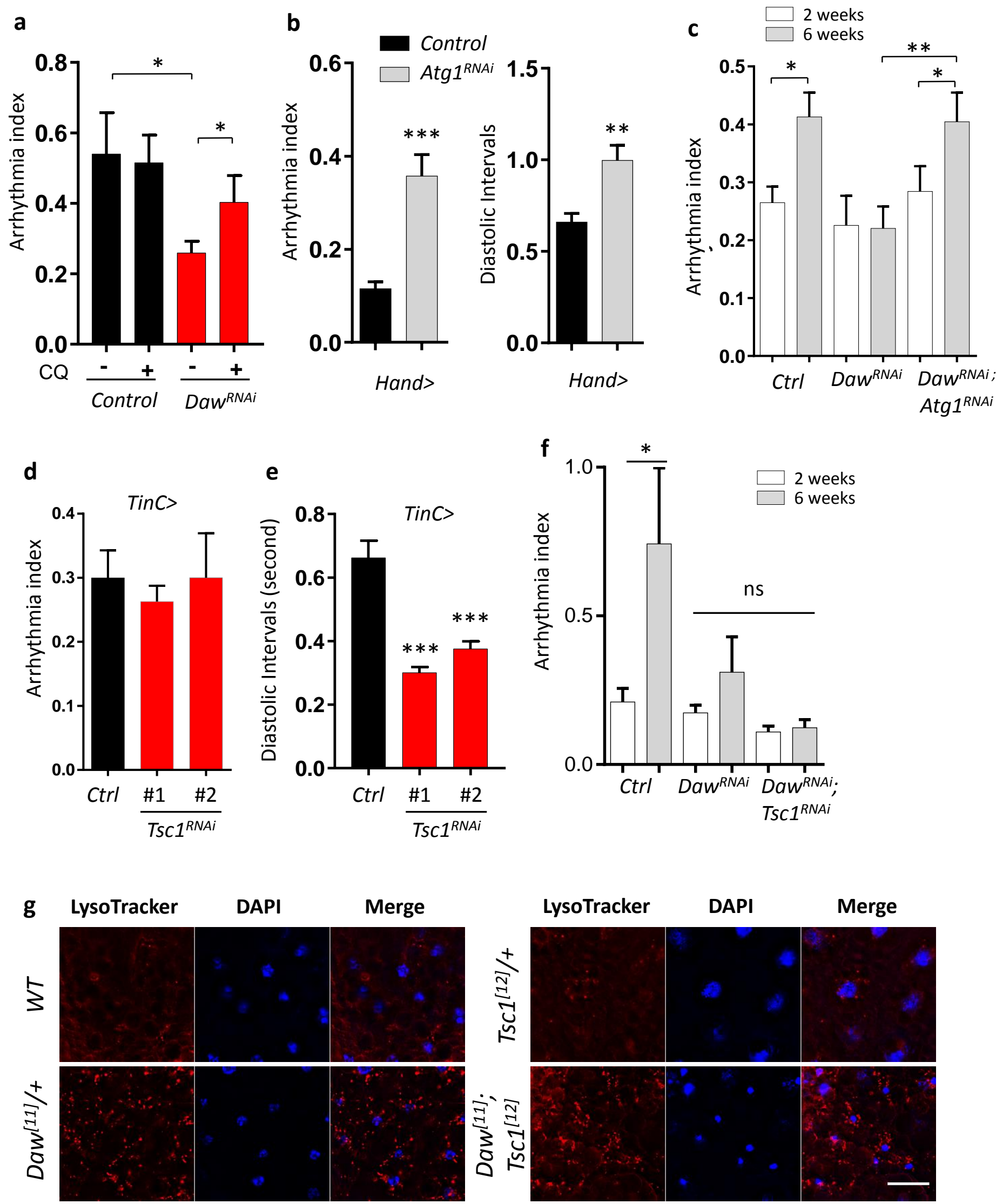
bioRxiv preprint doi: https://doi.org/10.1101/139360; this version posted October 1,2018 . The copyright holder for this preprint (which was not

Figure 5

a
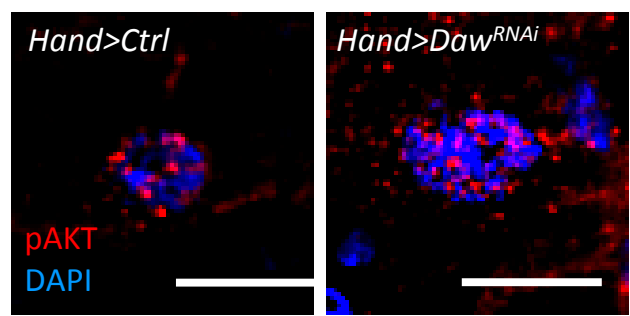

e

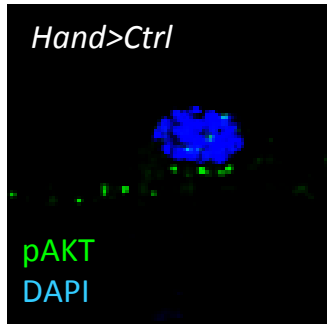

Hand $>$ Rictor $\mathrm{OE} \#$
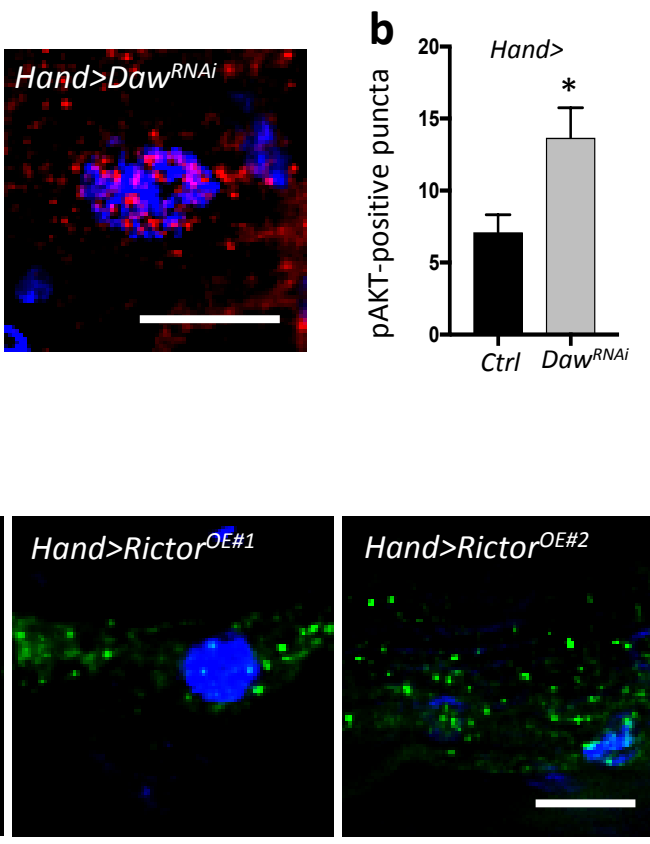

C

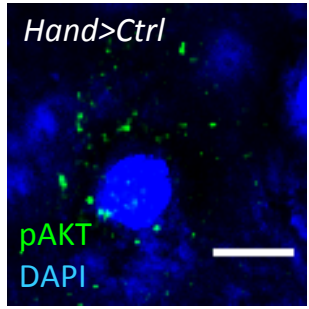

Hand $>$ Babo $^{A c t}$
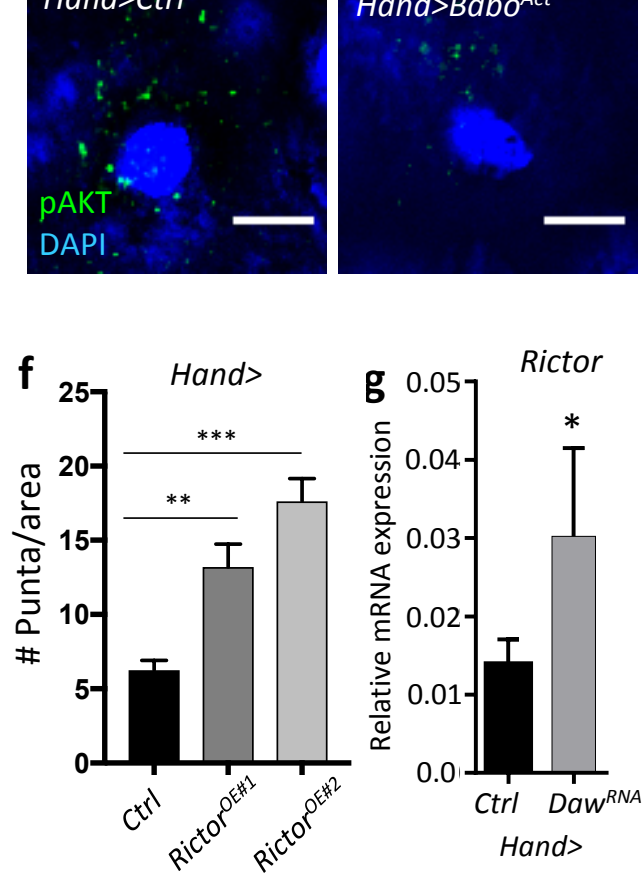

Hand $>$ Rictor $^{\mathrm{OE} 2}$
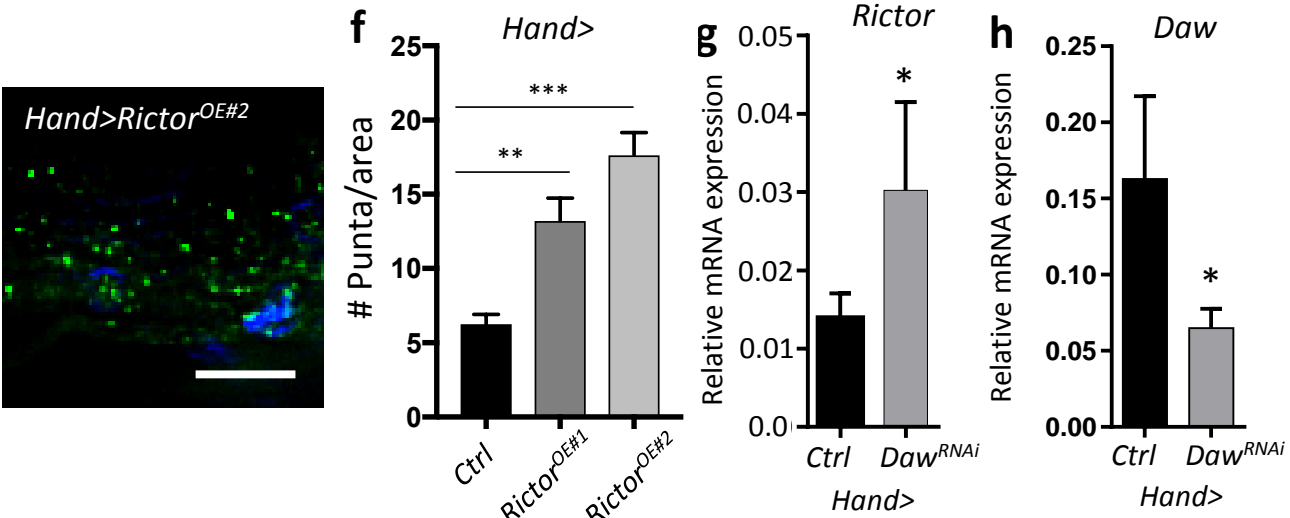

i

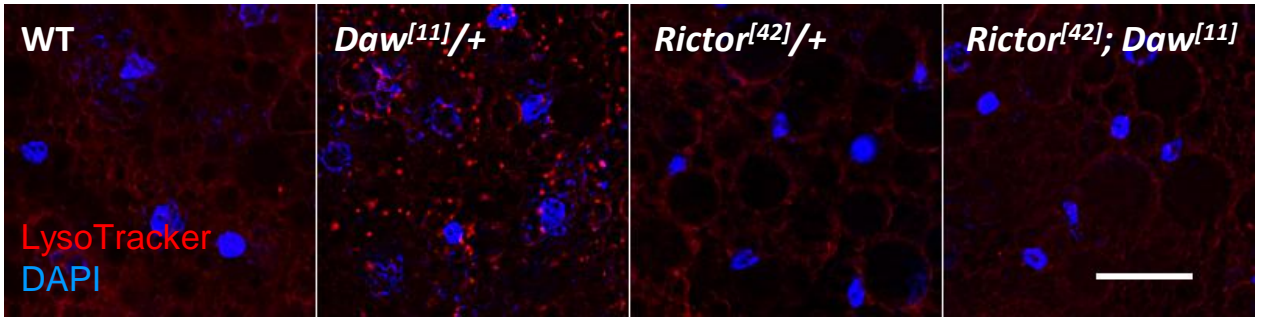

k

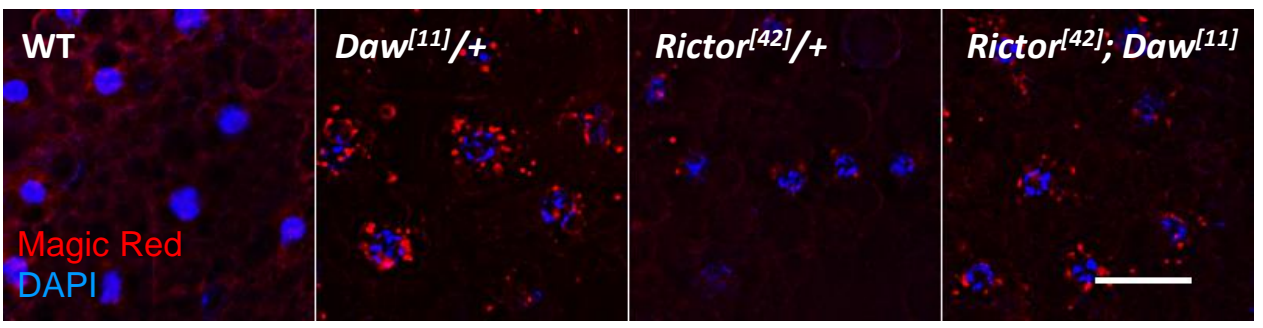

m

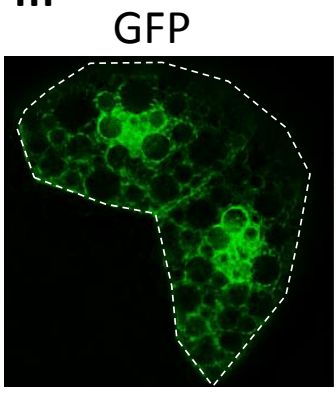

LysoTracker

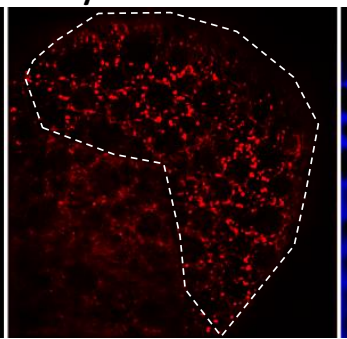

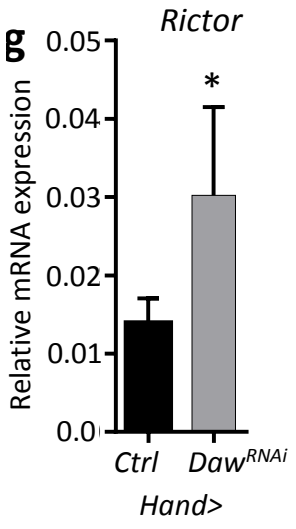

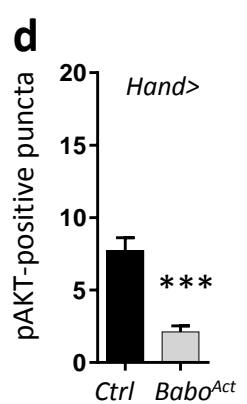

j
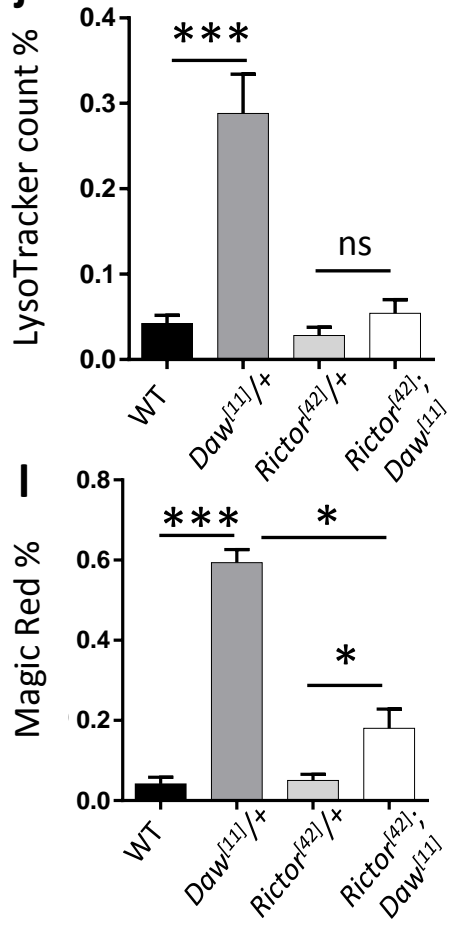


\section{Figure 6}
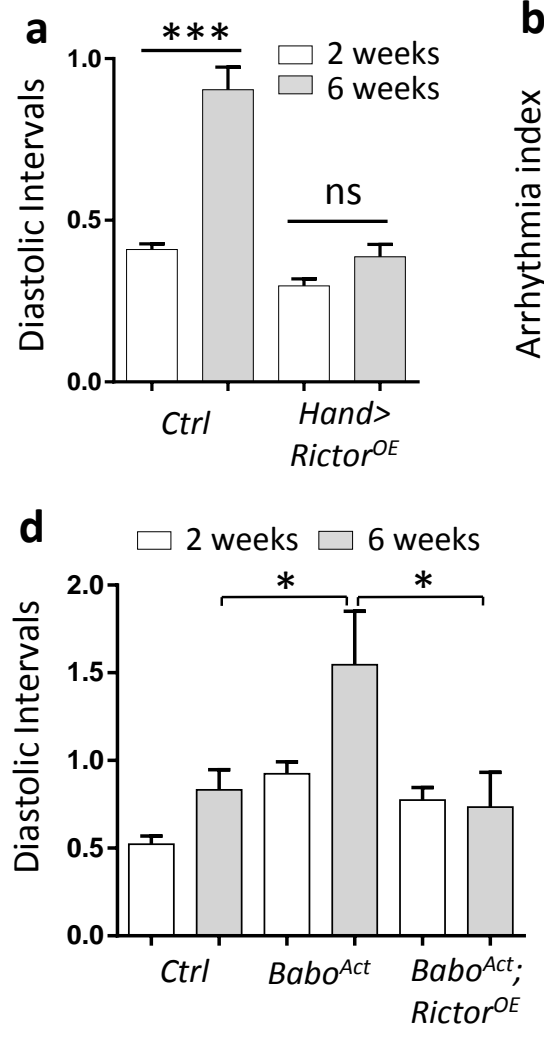

b
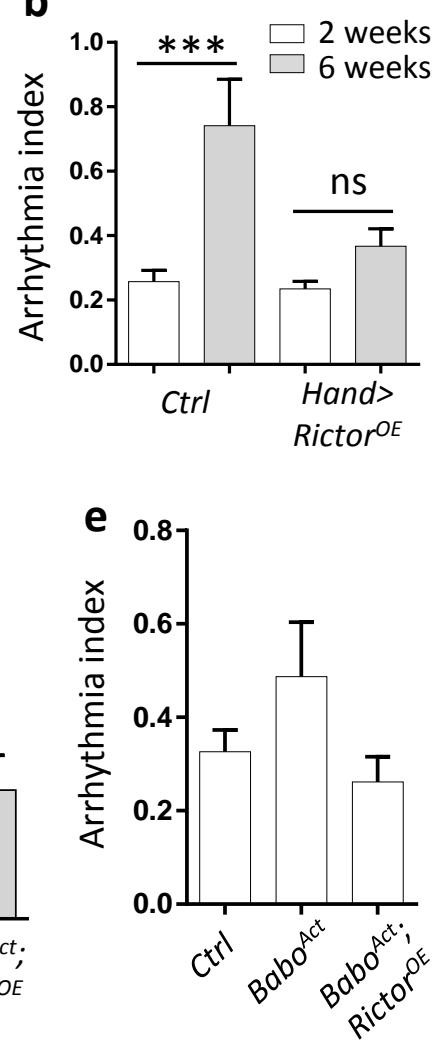

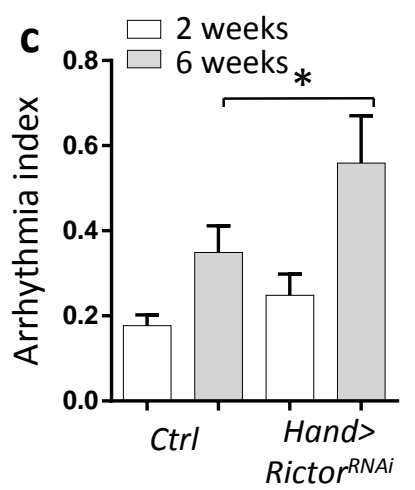

f

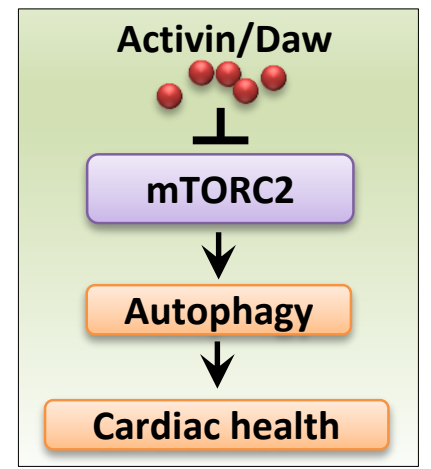


Figure 7
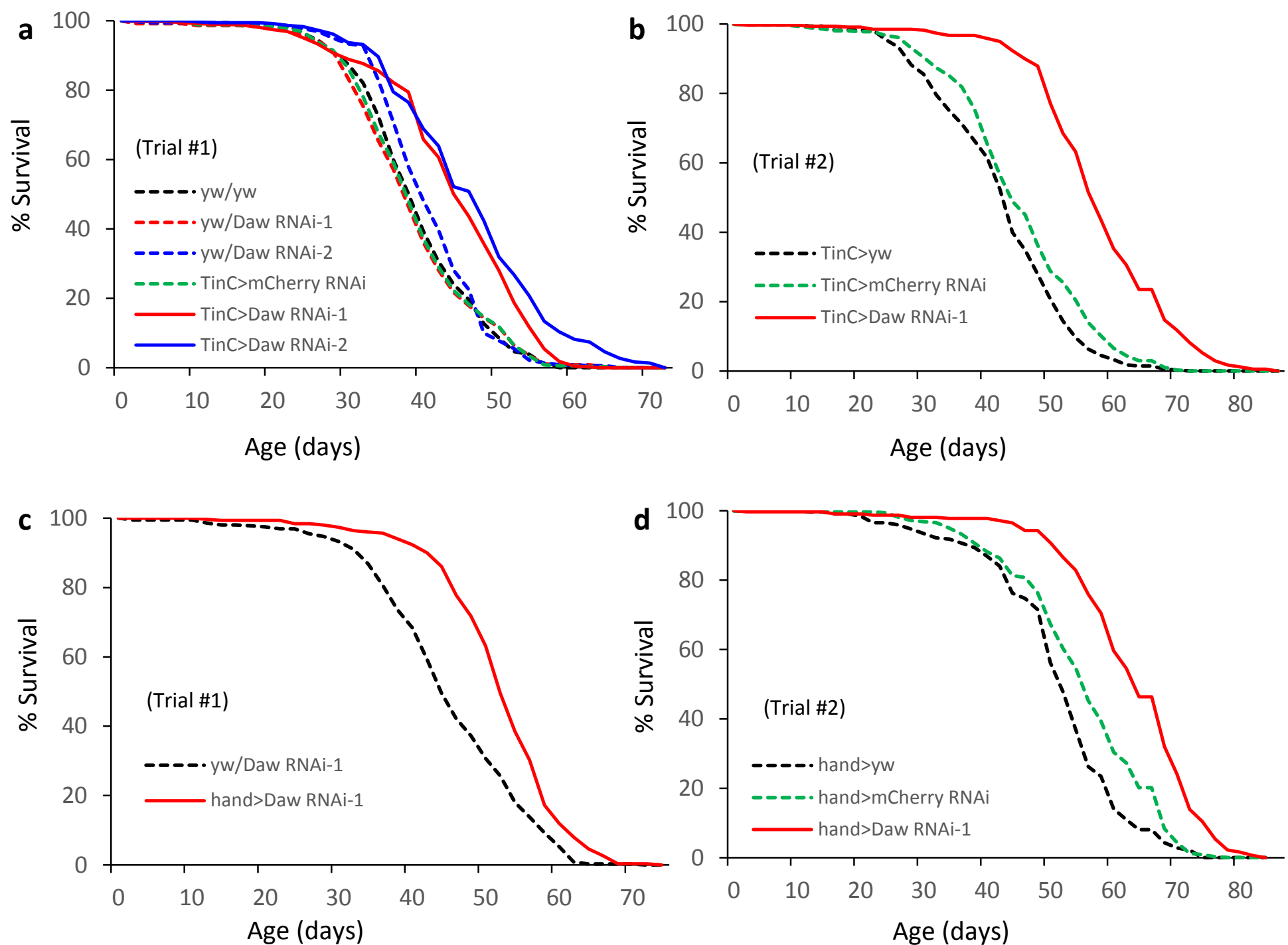

e

\begin{tabular}{|c|c|c|c|c|c|}
\hline Genotype & $\begin{array}{l}\text { Sample } \\
\text { size }\end{array}$ & $\begin{array}{c}\text { Mean lifespan } \\
\text { (days) }\end{array}$ & $\begin{array}{c}\text { Median lifespan } \\
\text { (days) }\end{array}$ & $\begin{array}{c}\text { Difference } \\
\text { RNAi vs. Control }\end{array}$ & $\begin{array}{c}\text { Prob. } \\
\text { (Log-Rank) }\end{array}$ \\
\hline \multicolumn{6}{|l|}{ Trial \#1 } \\
\hline $\operatorname{Tin} C>m C_{\text {Cherry }}^{R N A i}$ & 355 & 39.1 & 38 & / & / \\
\hline $\operatorname{Tin} C>D a w^{R N A i-1}$ & 389 & 44.3 & 46 & $21 \%$ & $<0.001$ \\
\hline Hand $>$ Daw $w^{R N A i-1}$ & 355 & 52.3 & 52 & $18 \%$ & $<0.001$ \\
\hline \multicolumn{6}{|l|}{ Trial \#2 } \\
\hline TinC $>m$ Cherry ${ }^{R N A i}$ & 364 & 45.3 & 44 & / & / \\
\hline Hand $>D a w^{R N A i-1}$ & 315 & 63.3 & 64 & $14 \%$ & $<0.001$ \\
\hline
\end{tabular}


Supplementary file

\section{Supplemental Figure S1}

a DmAtg8a hGABARAP

DmAtg $8 a$

hGABARAP

DmAtg $8 a$

hGABARAP
MKFQYKEEHAFEKRRAEGDKIRRKYPDRVPVIVEKAPKARIGDLDKKKYLVPSDLTVGQF MKFVYKEEHPFEKRRSEGEKIRKKYPDRVPVIVEKAPKARIGDLDKKKYLVPSDLTVGQF

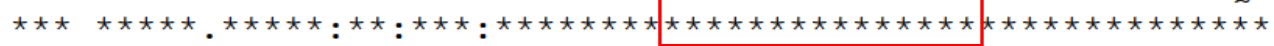

YFLIRKRIHLRPEDALFFFVNNVIPPTSATMGSLYQEHHEEDYFLYIAYSDENVYGMAKI YFLIRKRIHLRAEDALFFFVNNVIPPTSATMGQLYQEHHEEDFFLYIAYSDESVYGL--$\mathrm{N}$

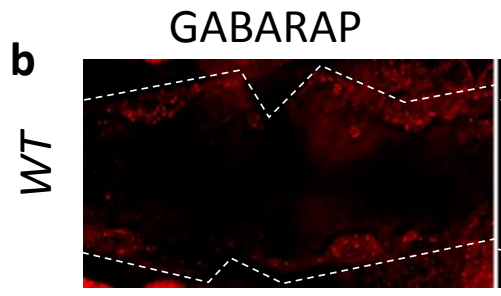

DAPI
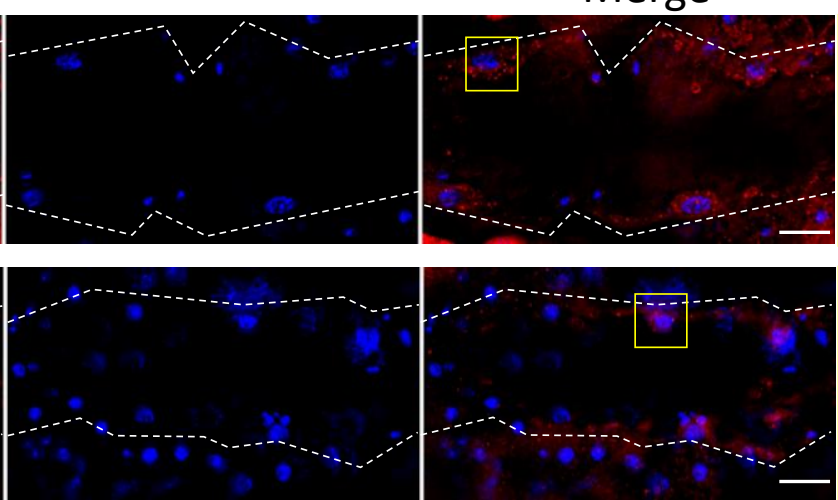

GABARAP DAPI S.
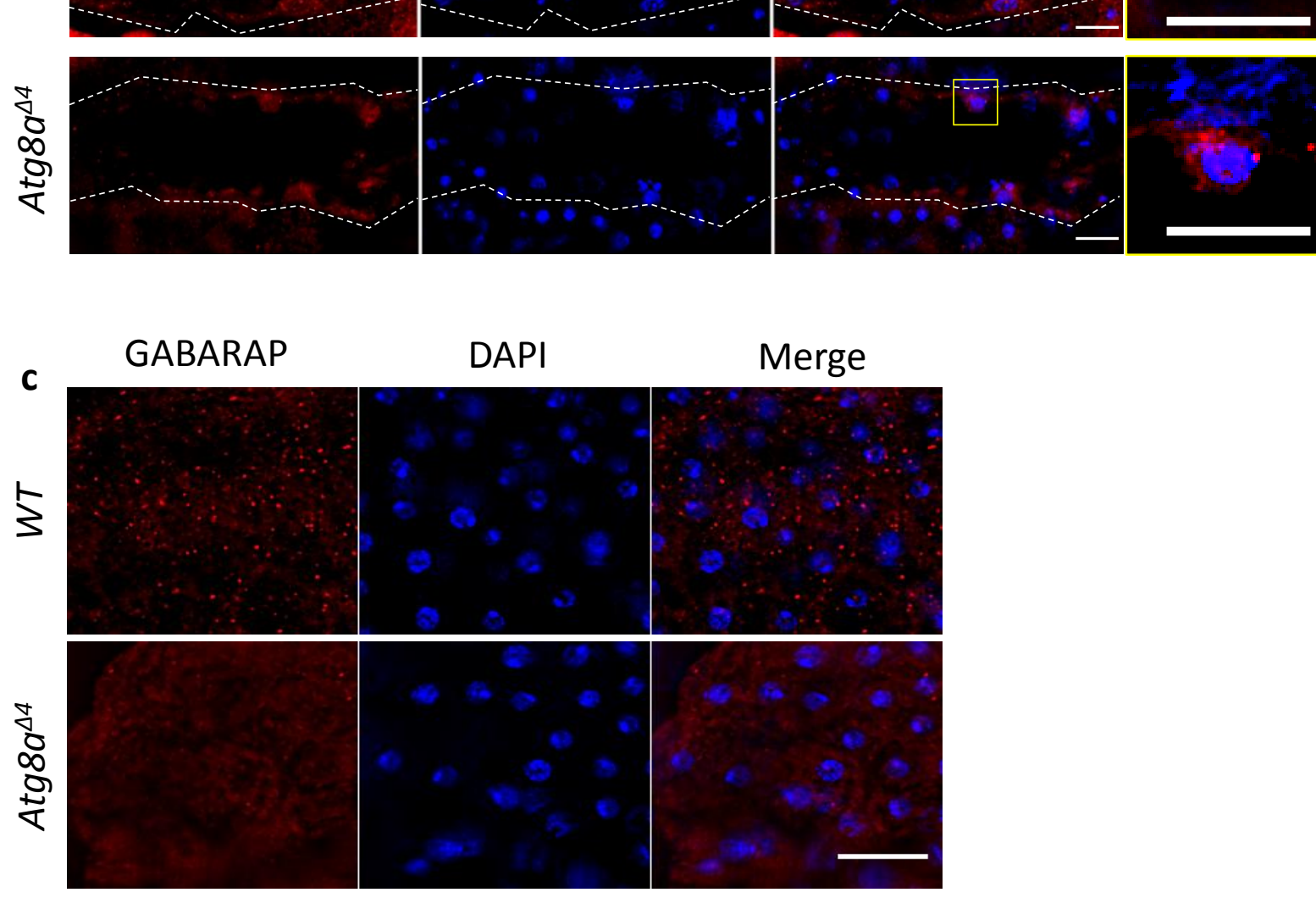

d

$\operatorname{Atg} 8 a^{\Delta 4}$

GABARAP

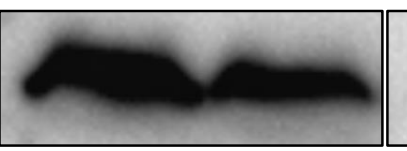


Supplementary file

Supplemental Figure S2

a
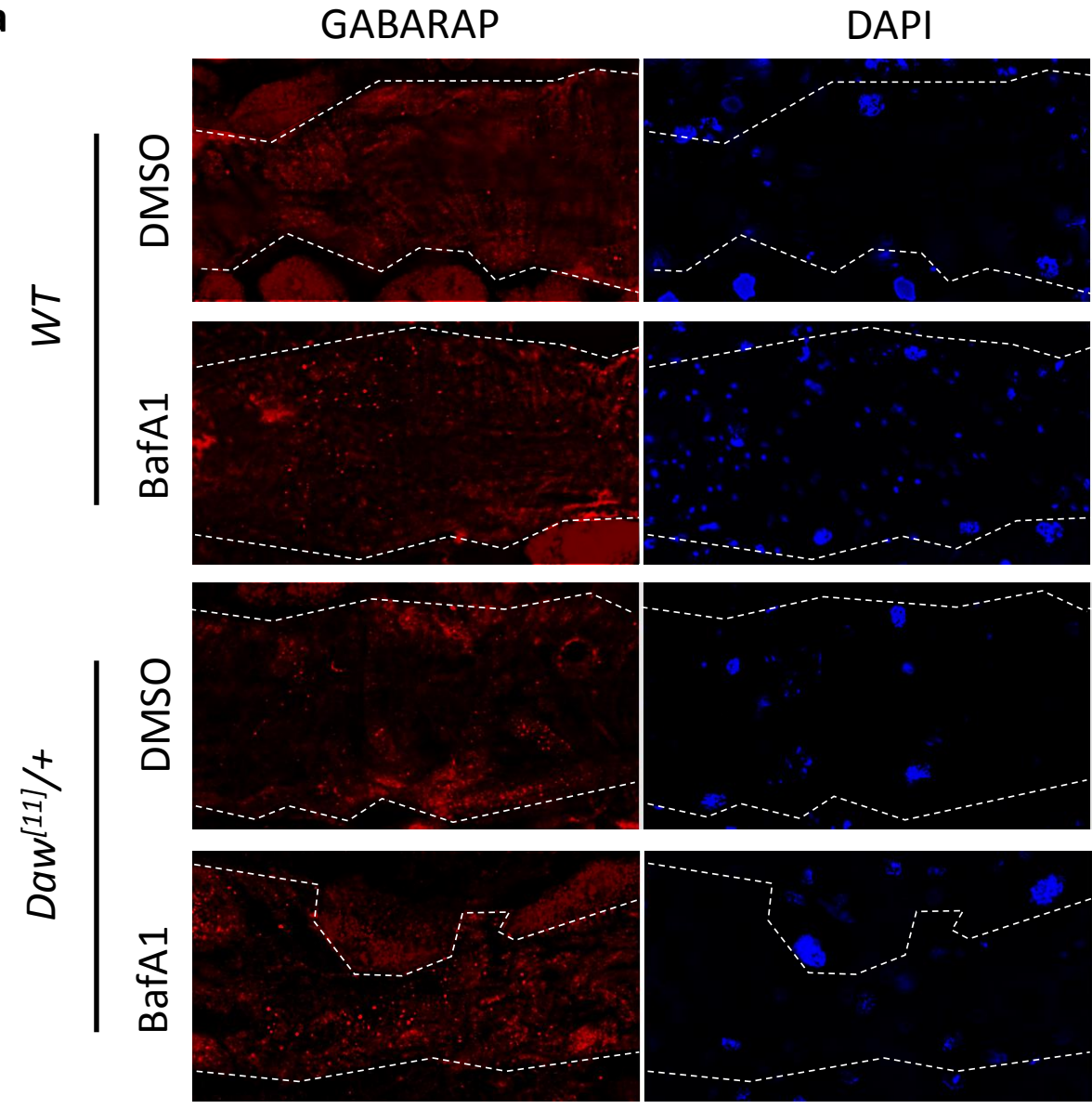

b

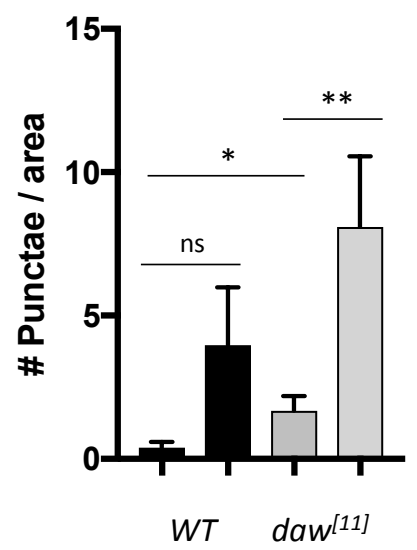

C

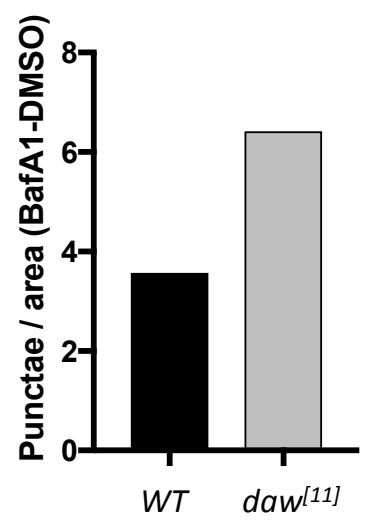




\section{Supplementary file}

Supplemental Figure S3

a

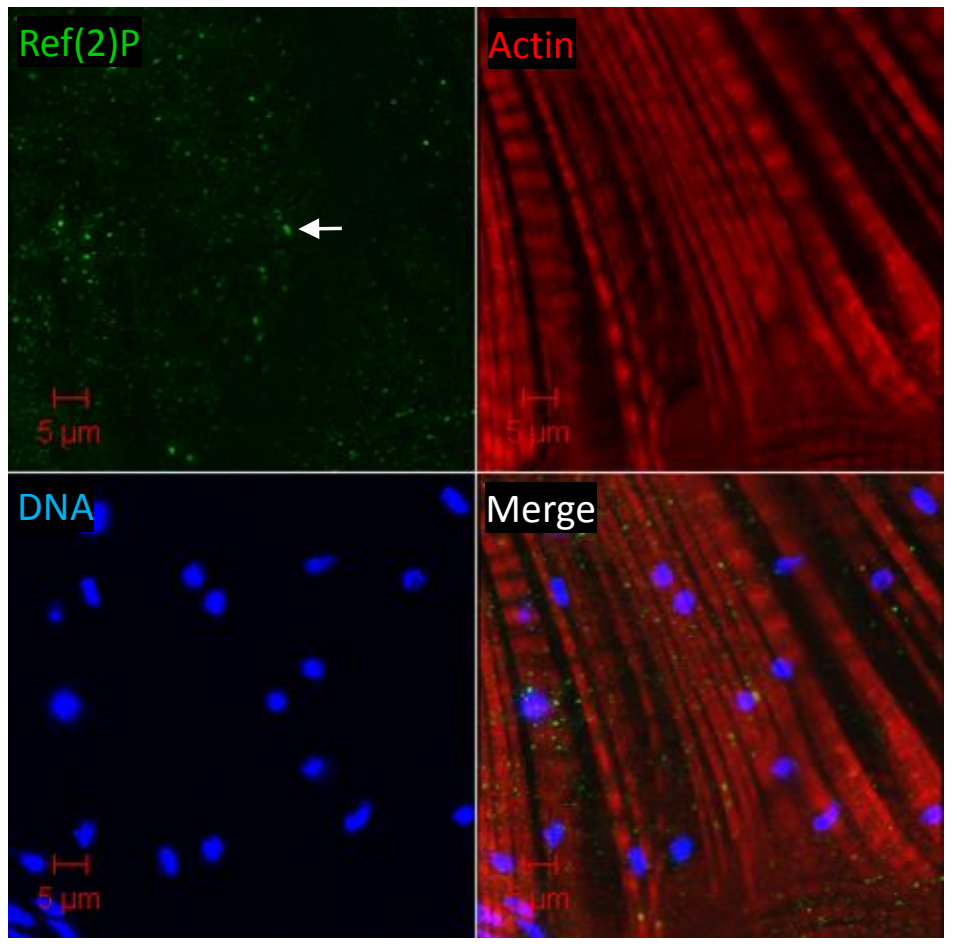

b

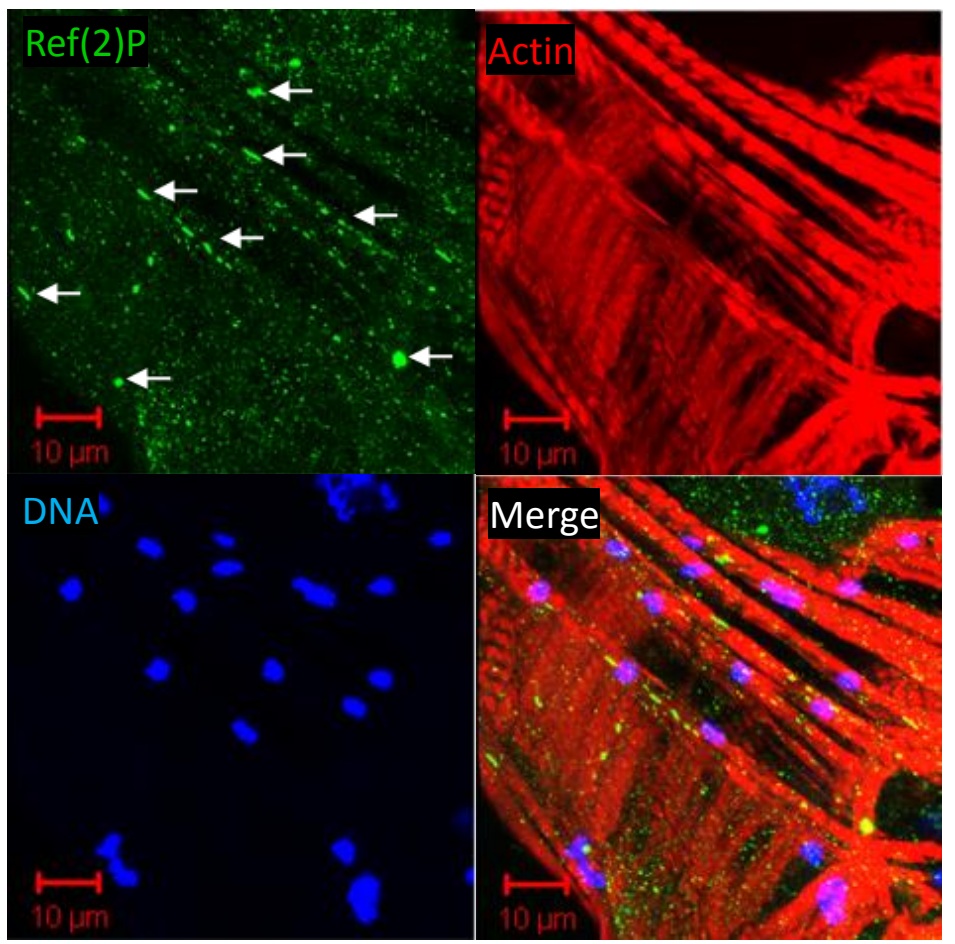

C

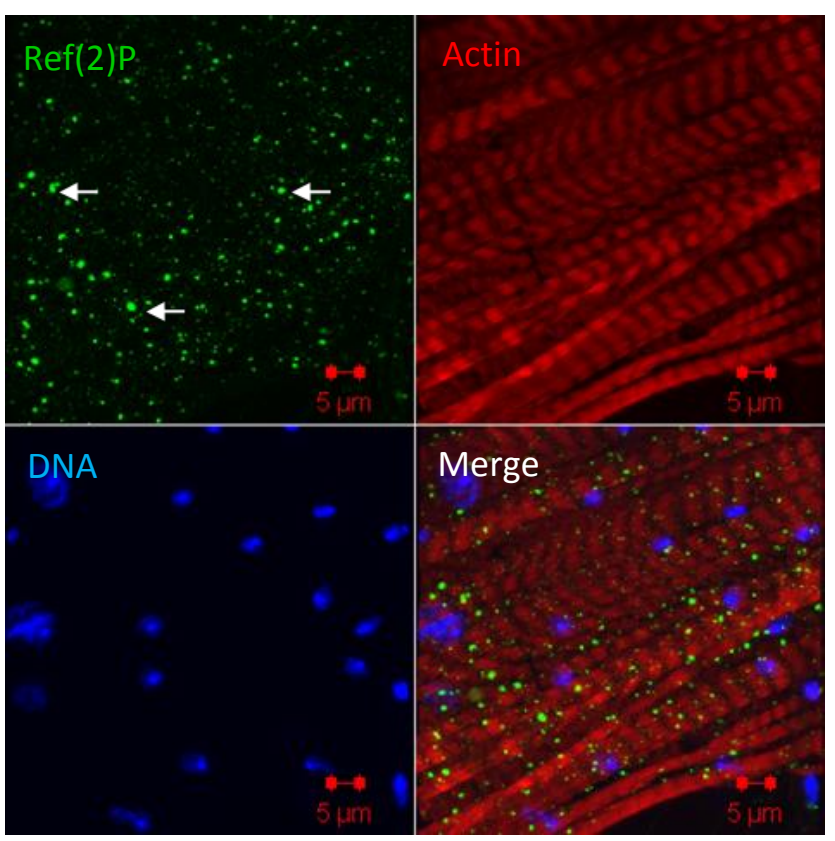




\section{Supplementary file}

Supplemental Figure S4

a

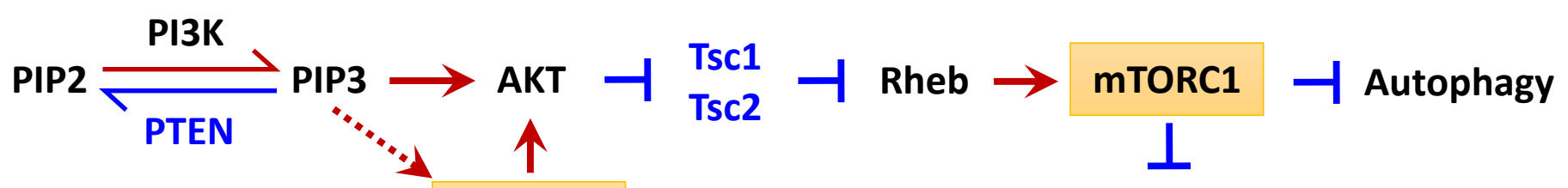

mTORC2

Reptor
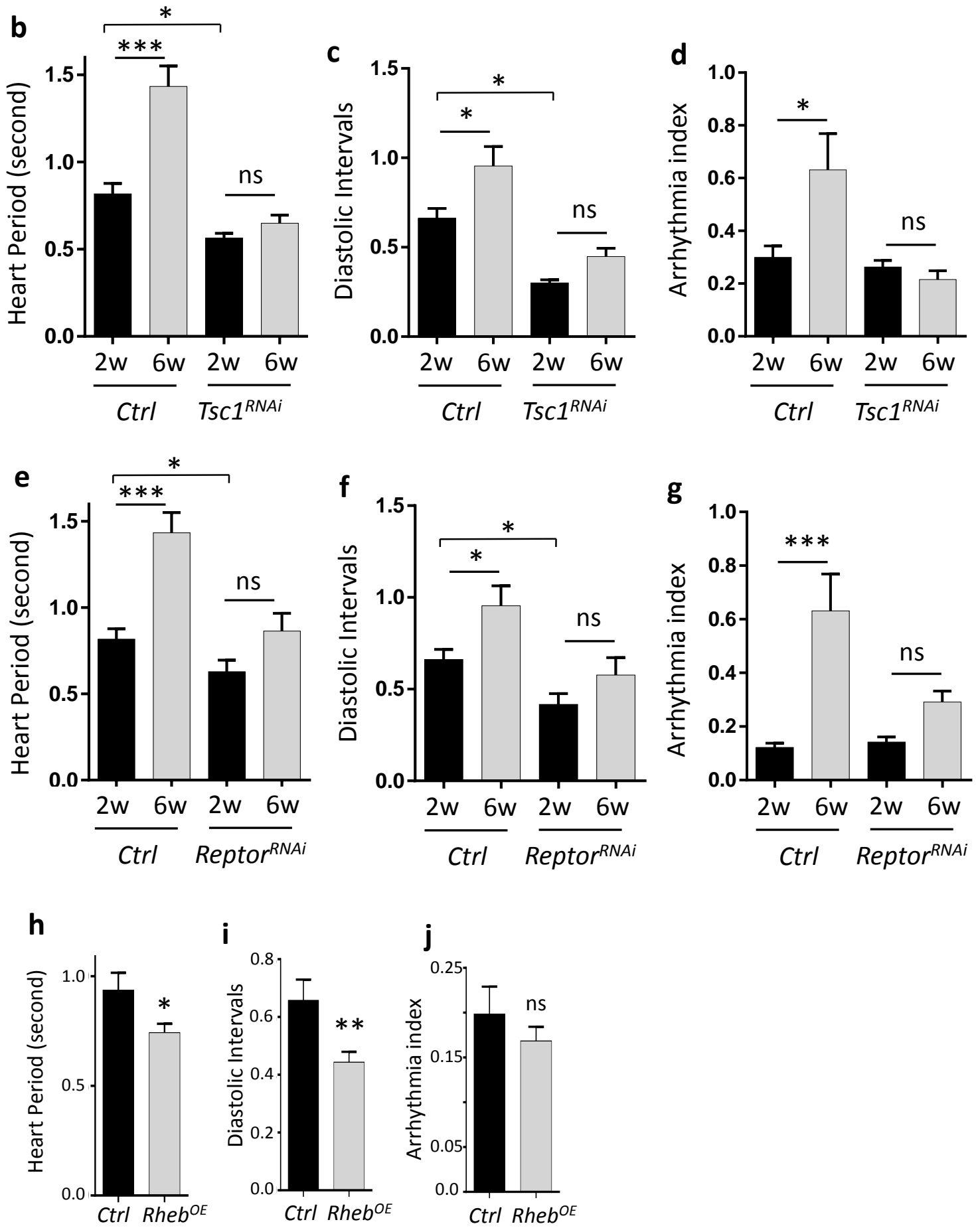
Supplementary file

Supplemental Figure S5

a

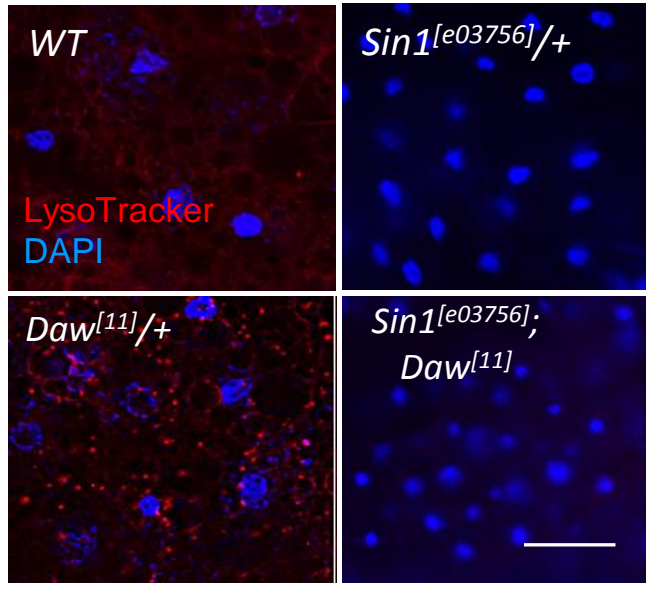

C

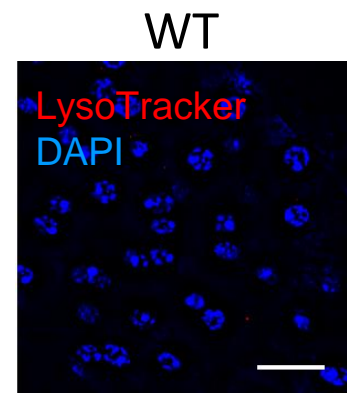

$\operatorname{Tsc} 1^{[12]} /+$
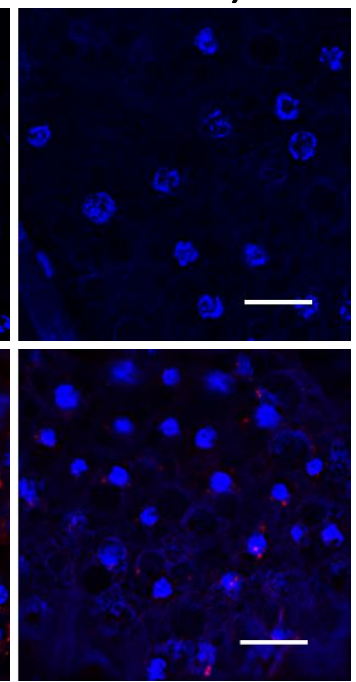

d

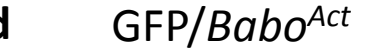

\begin{abstract}
LysoTracker
\end{abstract}

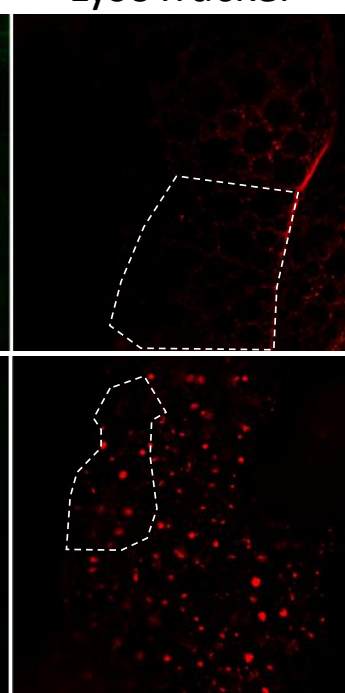

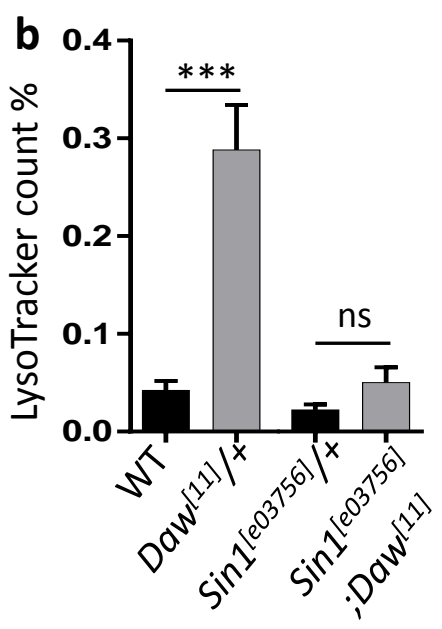

Rictor $^{[42]} /+$

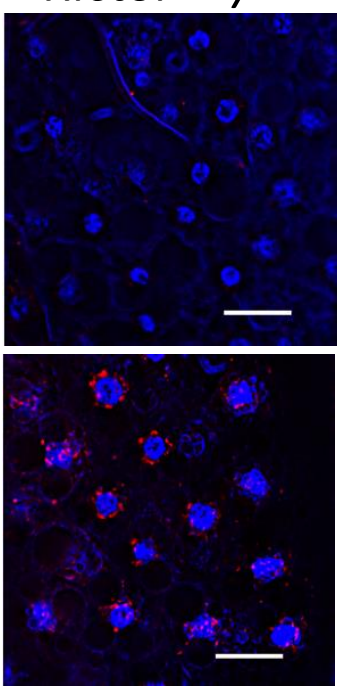

DAPI

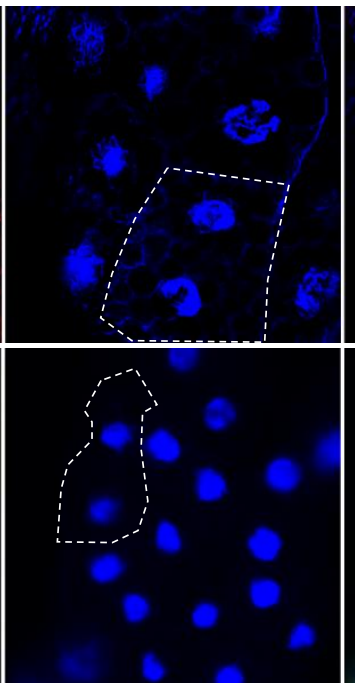

Merge

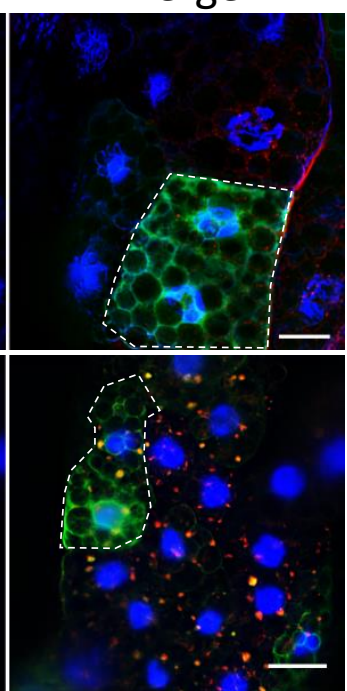


Supplementary Table S1.

\begin{tabular}{|l|l|l|l|}
\hline Primer name & Primer sequence (5' '-3') $^{\prime}$ & Symbol & FlyBase ID \\
\hline RpL32-f & AAGAAGCGCACCAAGCACTTCATC & RpL32 & FBgn0002626 \\
\hline RpL32-r & TCTGTTGTCGATACCCTTGGGCTT & RpL32 & FBgn0002626 \\
\hline Daw-f & TGAGCCACCTCATCCAAATCACCT & Daw & FBgn0031461 \\
\hline Daw-r & TCGATCACGATGAATGGCCGGTAA & Daw & FBgn0031461 \\
\hline Rictor-f & CCCAACTGGCTAGCGTTTAT & Rictor & FBgn0031006 \\
\hline Rictor-r & GGCCTTTGCTTTCGTTTGAG & Rictor & FBgn0031006 \\
\hline
\end{tabular}

